\title{
Bioactivity-Guided Synthesis Accelerates the Discovery of \\ 3-(Iso)quinolinyl-4-chromenones as Potent Fungicide Candidates
}

\section{Supporting Information}

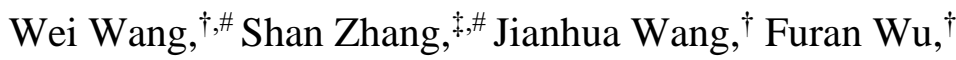

Tao Wang, ${ }^{*},+$ and Gong $\mathrm{Xu}^{*}, \dagger, \S$

†College of Plant Protection, Northwest A\&F University,

3 Taicheng Road, Yangling 712100, Shaanxi, China

*Key Laboratory of Applied Surface and Colloid Chemistry,

Ministry of Education, School of Chemistry and Chemical Engineering,

Shaanxi Normal University, No. 620 West Chang'an Avenue,

Xi'an 710119, Shaanxi Province, China.

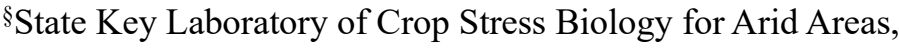

Key Laboratory of Botanical Pesticide R\&D in Shaanxi Province,

Shaanxi Key Laboratory of Natural Products \& Chemical Biology,

Yangling 712100, Shaanxi, China

${ }^{\#} \mathrm{~W}$. Wang and S. Zhang contributed equally.

* Corresponding authors

Prof. Dr. Tao Wang, orcid.org/0000-0002-1494-5297, E-mail: chemtao@snnu.edu.cn

Prof. Dr. Gong Xu, orcid.org/0000-0002-4305-4370, E-mail: gongxu@nwafu.edu.cn 


\section{List of Supplementary Materials}

Page 3 Biological Assay

Page 4-10 The Physical Data of Compounds 1a-h, 3-33

Page 11-31 $\quad{ }^{1} \mathrm{H}$ NMR and ${ }^{13} \mathrm{C}$ NMR Spectra for the Target Compounds 


\section{Biological Assay.}

The tested fungi were provided by the College of Plant Protection, Northwest A\&F University (Yangling, China). The tested compounds were dissolved in DMSO to give a $10 \mathrm{mg} / \mathrm{mL}$ stock solution before mixing with PDA. The media containing compounds at a concentration of $50 \mathrm{mg} / \mathrm{L}$ were then poured into sterilized Petri dishes for primary screening. After $24-72 \mathrm{~h}$ at $28{ }^{\circ} \mathrm{C}$, the colony diameter of each strain was measured. Percentage inhibition rate was calculated as (C-A)/(CB) $\times 100 \%$, where A represents the colony diameter in the Petri dishes with tested compounds, B represents the diameter of mycelial disc, and $\mathrm{C}$ is the mean colony diameter in control Petri dishes.

Quercetin, chlorothalonil, tebuconazole, procymidone and boscalid were selected as the positive control. Each treatment was performed three times. The inhibition rate of the potent compounds was further tested, and the corresponding $\mathrm{EC}_{50}$ values were calculated by SPSS 10.0. 


\section{The Physical Data of Intermediates 1a-h and Compounds 3-33}

Compounds 1a, ${ }^{1} 1 \mathrm{~b},{ }^{2} 1 \mathrm{c},{ }^{1} 1 \mathrm{~d},{ }^{3} 1 \mathrm{e},{ }^{4} 1 \mathrm{f},{ }^{3} 1 \mathrm{gg},{ }^{5} 1 \mathrm{~h},{ }^{5} 3,{ }^{5} 8,{ }^{5} 11,{ }^{5}$ and $23-33$ were known compounds which were prepared by following the literature procedures. ${ }^{5}$

\section{1-(2-Hydroxyphenyl)-3-phenylprop-2-yn-1-one (1a)}

Yellow solid, 70\% yield; $\mathrm{mp}=64.2-65.3{ }^{\circ} \mathrm{C} ;{ }^{1} \mathrm{H}$ NMR $(400 \mathrm{MHz}$, Chloroform- $d$ ) $\delta 11.76(\mathrm{~s}, 1 \mathrm{H})$, 8.15-8.10 (m, $1 \mathrm{H}), 7.69(\mathrm{~d}, J=7.1 \mathrm{~Hz}, 2 \mathrm{H}), 7.52(\mathrm{q}, J=7.7 \mathrm{~Hz}, 2 \mathrm{H}), 7.44(\mathrm{~d}, J=14.8 \mathrm{~Hz}, 2 \mathrm{H}), 6.99$ $(\mathrm{d}, J=14.8 \mathrm{~Hz}, 2 \mathrm{H})$.

1-(2-Hydroxyphenyl)-3-(p-tolyl)prop-2-yn-1-one (1b)

Yellow solid, 77\% yield; $\mathrm{mp}=84.8-85.6{ }^{\circ} \mathrm{C} ;{ }^{1} \mathrm{H}$ NMR $(400 \mathrm{MHz}$, Chloroform- $d) \delta 11.78(\mathrm{~s}, 1 \mathrm{H})$, 8.11 (s, $1 \mathrm{H}), 7.61-7.44$ (m, $3 \mathrm{H}), 7.30$ (s, $2 \mathrm{H}), 6.99$ (d, J = 7.7 Hz, $2 \mathrm{H}), 2.38$ (s, $3 \mathrm{H})$.

1-(2-Hydroxyphenyl)-3-(4-methoxyphenyl)prop-2-yn-1-one (1c)

Yellow solid, $86 \%$ yield; $\mathrm{mp}=88.4-89.5{ }^{\circ} \mathrm{C} ;{ }^{1} \mathrm{H}$ NMR $(400 \mathrm{MHz}$, Chloroform- $d$ ) $\delta 11.84(\mathrm{~s}, 1 \mathrm{H})$, $8.11(\mathrm{~d}, J=8.3 \mathrm{~Hz}, 1 \mathrm{H}), 7.65(\mathrm{~d}, J=8.8 \mathrm{~Hz}, 2 \mathrm{H}), 7.51(\mathrm{t}, J=7.8 \mathrm{~Hz}, 1 \mathrm{H}), 6.97(\mathrm{dd}, J=20.3,8.3 \mathrm{~Hz}$, $4 \mathrm{H}), 3.86$ (s, $3 \mathrm{H})$.

3-(4-Fluorophenyl)-1-(2-hydroxyphenyl)prop-2-yn-1-one (1d)

Yellow solid, $83 \%$ yield; mp $=109.3-110.2{ }^{\circ} \mathrm{C} ;{ }^{1} \mathrm{H}$ NMR $(400 \mathrm{MHz}$, Chloroform- $d) \delta 11.72(\mathrm{~s}, 1$ H), $8.08(\mathrm{~d}, J=7.8 \mathrm{~Hz}, 1 \mathrm{H}), 7.74-7.64(\mathrm{~m}, 2 \mathrm{H}), 7.52(\mathrm{t}, J=7.5 \mathrm{~Hz}, 1 \mathrm{H}), 7.13(\mathrm{t}, J=8.4 \mathrm{~Hz}, 2 \mathrm{H})$, $6.98(\mathrm{t}, J=7.4 \mathrm{~Hz}, 2 \mathrm{H})$.

3-(4-Chlorophenyl)-1-(2-hydroxyphenyl)prop-2-yn-1-one (1e)

Yellow solid, $72 \%$ yield; $\mathrm{mp}=81.5-82.6{ }^{\circ} \mathrm{C} ;{ }^{1} \mathrm{H}$ NMR $(400 \mathrm{MHz}$, Chloroform- $d) \delta 11.69(\mathrm{~s}, 1 \mathrm{H})$, $8.09(\mathrm{~d}, J=7.9 \mathrm{~Hz}, 1 \mathrm{H}), 7.63(\mathrm{~d}, J=8.3 \mathrm{~Hz}, 2 \mathrm{H}), 7.54(\mathrm{t}, J=7.8 \mathrm{~Hz}, 1 \mathrm{H}), 7.43(\mathrm{~d}, J=8.4 \mathrm{~Hz}, 2 \mathrm{H})$, $7.00(\mathrm{t}, J=8.5 \mathrm{~Hz}, 2 \mathrm{H})$. 
1-(2-Hydroxyphenyl)-3-(4-(trifluoromethyl)phenyl)prop-2-yn-1-one (1f)

Yellow solid, $75 \%$ yield; mp $=80.2-81.7^{\circ} \mathrm{C} ;{ }^{1} \mathrm{H}$ NMR $(400 \mathrm{MHz}$, Chloroform- $d) \delta 11.61(\mathrm{~s}, 1$ H), $8.09(\mathrm{~d}, J=7.7 \mathrm{~Hz}, 1 \mathrm{H}), 7.80(\mathrm{~d}, J=8.1 \mathrm{~Hz}, 2 \mathrm{H}), 7.70(\mathrm{~d}, J=8.2 \mathrm{~Hz}, 2 \mathrm{H}), 7.55$ (t, $J=7.7$ $\mathrm{Hz}, 1 \mathrm{H}), 7.00$ (s, $2 \mathrm{H})$.

1-(2-Hydroxyphenyl)-3-(m-tolyl)prop-2-yn-1-one (1g)

Yellow solid, $82 \%$ yield; $\mathrm{mp}=55.0-55.9^{\circ} \mathrm{C} ;{ }^{1} \mathrm{H}$ NMR $(400 \mathrm{MHz}$, Chloroform- $d) \delta 11.77(\mathrm{~s}, 1$ H), $8.13(\mathrm{~d}, J=8.3 \mathrm{~Hz}, 1 \mathrm{H}), 7.57-7.49(\mathrm{~m}, 3 \mathrm{H}), 7.33(\mathrm{~d}, J=5.2 \mathrm{~Hz}, 2 \mathrm{H}), 7.00(\mathrm{t}, J=7.7 \mathrm{~Hz}, 2$ H), $2.39(\mathrm{~s}, 3 \mathrm{H})$.

3-([1,1'-Biphenyl]-4-yl)-1-(2-hydroxyphenyl)prop-2-yn-1-one (1h)

Yellow solid, 50\% yield; $\mathrm{mp}=110.0-112.0{ }^{\circ} \mathrm{C} ;{ }^{1} \mathrm{H}$ NMR $\left(400 \mathrm{MHz}, \mathrm{CDCl}_{3}\right): \delta=11.78(\mathrm{~s}, 1 \mathrm{H})$, 8.17-8.15 (m, $1 \mathrm{H})$, 7.79-7.76 (m, 2H), 7.69-7.67 (m, $2 \mathrm{H})$, 7.64-7.62 (m, $2 \mathrm{H})$, 7.56-7.52 (m, $1 \mathrm{H})$, 7.50-7.47 (m, 2 H), 7.43-7.39 (m, $1 \mathrm{H}), 7.03-7.00$ (m, $2 \mathrm{H})$.

\section{3-(5-Methylquinolin-2-yl)-2-phenyl-4H-chromen-4-one (4)}

Brown solid, 71\% yield; $\mathrm{mp}=251.1-252.4{ }^{\circ} \mathrm{C} ;{ }^{1} \mathrm{H}$ NMR $\left(600 \mathrm{MHz}, \mathrm{CDCl}_{3}\right): \delta=8.33-8.30(\mathrm{~m}, 2$ H ), 7.81-7.79 (m, $1 \mathrm{H}), 7.73-7.71(\mathrm{~m}, 1 \mathrm{H}), 7.58-7.56(\mathrm{~m}, 1 \mathrm{H})$, 7.54-7.52 (m, $2 \mathrm{H})$, 7.45-7.42 (m, $3 \mathrm{H}), 7.36-7.34(\mathrm{~m}, 1 \mathrm{H}), 7.30-7.27$ (m, $1 \mathrm{H}), 7.21-7.19(\mathrm{~m}, 2 \mathrm{H}), 2.68(\mathrm{~s}, 3 \mathrm{H}) ;{ }^{13} \mathrm{C} \mathrm{NMR}(150 \mathrm{MHz}$, $\left.\mathrm{CDCl}_{3}\right): \delta=177.5,163.3,156.2,153.3,148.5,134.5,134.0,133.0,132.8,130.4,129.4,129.2,128.2$ 127.9, 127.3, 126.6, 126.3, 125.4, 123.9, 123.9, 123.2, 118.1, 18.8; HRMS (ESI): calculated for $\mathrm{C}_{25} \mathrm{H}_{17} \mathrm{NO}_{2}[\mathrm{M}+\mathrm{H}]^{+}: 364.1332$, found 364.1338.

3-(5-Fluoroquinolin-2-yl)-2-phenyl-4H-chromen-4-one (5)

Brown solid, $65 \%$ yield; $\mathrm{mp}=211.2-211.6{ }^{\circ} \mathrm{C} ;{ }^{1} \mathrm{H}$ NMR $\left(600 \mathrm{MHz}, \mathrm{CDCl}_{3}\right): \delta=8.43-8.41(\mathrm{~m}, 1$ H), 8.32-8.30 (m, $1 \mathrm{H})$, 7.76-7.72 (m, $2 \mathrm{H})$, 7.59-7.54 (m, $3 \mathrm{H})$, 7.47-7.45 (m, $1 \mathrm{H})$, 7.41-7.40 (m, 2 H), 7.32-7.30 (m, $1 \mathrm{H}), 7.23-7.18$ (m, $\left.3 \mathrm{H}) ;{ }^{13} \mathrm{C} \mathrm{NMR} \mathrm{(150} \mathrm{MHz,} \mathrm{CDCl}_{3}\right): \delta=177.3,163.6,157.9$, $156.3,154.9,149.0,134.1,132.8,130.6,129.5,129.4,129.0,128.3,126.4,125.5,125.47,124.5$, 123.9, 123.0, 118.2, 118.0, 110.4; HRMS (ESI): calculated for $\mathrm{C}_{24} \mathrm{H}_{14} \mathrm{FNO}_{2}[\mathrm{M}+\mathrm{H}]^{+}$: 368.1081 , found 368.1086 . 
3-(5-Chloroquinolin-2-yl)-2-phenyl-4H-chromen-4-one (6)

Brown solid, $57 \%$ yield; $\mathrm{mp}=203.6-204.8{ }^{\circ} \mathrm{C} ;{ }^{1} \mathrm{H}$ NMR $\left(600 \mathrm{MHz}, \mathrm{CDCl}_{3}\right): \delta=8.57-8.56(\mathrm{~m}, 1$ H ), 8.32-8.30 (m, $1 \mathrm{H})$, 7.87-7.86 (m, $1 \mathrm{H})$, 7.75-7.72 (m, $1 \mathrm{H})$, 7.61-7.54 (m, $4 \mathrm{H})$, 7.47-7.44 (m, $1 \mathrm{H}), 7.40-7.39$ (m, $2 \mathrm{H}), 7.32-7.29$ (m, $1 \mathrm{H}), 7.23-7.20$ (m, $2 \mathrm{H}) ;{ }^{13} \mathrm{C}$ NMR (150 MHz, $\left.\mathrm{CDCl}_{3}\right): \delta$ $=177.3,163.7,156.2,154.7,148.8,134.2,133.1,132.8,131.3,130.6,129.4,129.2,128.8,128.3$, $126.9,126.4,125.5,125.4,125.2,123.8,122.7,118.2$; HRMS (ESI): calculated for $\mathrm{C}_{24} \mathrm{H}_{14} \mathrm{ClNO}_{2}[\mathrm{M}+\mathrm{Na}]^{+}:$406.0605, found 406.0622 .

\section{3-(5-Bromoquinolin-2-yl)-2-phenyl-4H-chromen-4-one (7)}

Brown solid, $65 \%$ yield; $\mathrm{mp}=201.2-202.6{ }^{\circ} \mathrm{C} ;{ }^{1} \mathrm{H}$ NMR $\left(600 \mathrm{MHz}, \mathrm{CDCl}_{3}\right): \delta=8.53-8.51(\mathrm{~m}, 1$ H ), 8.31-8.30 (m, $1 \mathrm{H})$, 7.91-7.89 (m, $1 \mathrm{H}), 7.80-7.79(\mathrm{~m}, 1 \mathrm{H})$, 7.74-7.72 (m, $1 \mathrm{H})$, 7.61-7.57 (m, $2 \mathrm{H})$, 7.50-7.44 (m, $2 \mathrm{H}), 7.40-7.38$ (m, $2 \mathrm{H}), 7.32-7.29$ (m, $1 \mathrm{H}), 7.23-7.20$ (m, $2 \mathrm{H}) ;{ }^{13} \mathrm{C}$ NMR $\left(150 \mathrm{MHz}, \mathrm{CDCl}_{3}\right): \delta=177.3,163.7,156.2,154.8,148.9,135.7,134.2,132.8,130.6,129.7,129.5$, 129.4, 128.3, 126.7, 126.3, 125.5, 125.5, 123.8, 122.6, 121.8, 118.1; HRMS (ESI): calculated for $\mathrm{C}_{24} \mathrm{H}_{14} \mathrm{BrNO}_{2}[\mathrm{M}+\mathrm{H}]^{+}:$428.0281, found 428.0286.

\section{3-(6-Methylquinolin-2-yl)-2-phenyl-4H-chromen-4-one (9)}

Brown solid, 70\% yield; $\mathrm{mp}=176.8-178.2{ }^{\circ} \mathrm{C} ;{ }^{1} \mathrm{H}$ NMR $\left(600 \mathrm{MHz}, \mathrm{CDCl}_{3}\right): \delta=8.30-8.29(\mathrm{~m}, 1$ H ), 8.04-8.02 (m, 1 H), 7.84-7.83 (m, 1 H), 7.70-7.67 (m, 1 H), 7.55-7.54 (m, 2 H), 7.46-7.39 (m, $5 \mathrm{H}), 7.25-7.24(\mathrm{~m}, 1 \mathrm{H}), 7.18-7.15(\mathrm{~m}, 2 \mathrm{H}), 2.49$ (s, $3 \mathrm{H}) ;{ }^{13} \mathrm{C} \mathrm{NMR}\left(150 \mathrm{MHz}, \mathrm{CDCl}_{3}\right): \delta=177.4$, $163.2,156.2,152.7,146.8,136.6,135.5,133.9,132.9,131.7,130.3,129.3,129.2,128.1,127.2$, 126.4, 126.3, 125.3, 124.2, 123.8, 123.2, 118.0, 21.6; HRMS (ESI): calculated for $\mathrm{C}_{25} \mathrm{H}_{17} \mathrm{NO}_{3}$ $[\mathrm{M}+\mathrm{H}]^{+}:$384.1332, found 384.1335.

\section{3-(6-Fluoroquinolin-2-yl)-2-phenyl-4H-chromen-4-one (10)}

Brown solid, $65 \%$ yield; $\mathrm{mp}=148.2-150.1{ }^{\circ} \mathrm{C} ;{ }^{1} \mathrm{H}$ NMR $\left(600 \mathrm{MHz}, \mathrm{CDCl}_{3}\right): \delta=8.31-8.30(\mathrm{~m}, 1$ H ), 8.10-8.08 (m, $1 \mathrm{H})$, 7.94-7.92 (m, $1 \mathrm{H}), 7.73-7.71(\mathrm{~m}, 1 \mathrm{H})$, 7.58-7.56 (m, $1 \mathrm{H})$, 7.51-7.50 (m, $1 \mathrm{H}), 7.46-7.39$ (m, $5 \mathrm{H}), 7.30-7.28$ (m, $1 \mathrm{H}), 7.21-7.19$ (m, $2 \mathrm{H}) ;{ }^{13} \mathrm{C} \mathrm{NMR}\left(150 \mathrm{MHz}, \mathrm{CDCl}_{3}\right): \delta$ $=177.4,163.5,160.7(\mathrm{~d}, J=247.1 \mathrm{~Hz}), 156.2,153.2(\mathrm{~d}, J=2.8 \mathrm{~Hz}), 145.3,135.6(\mathrm{~d}, J=5.3 \mathrm{~Hz})$, 
134.1, 132.9, $132.1(\mathrm{~d}, J=9.2 \mathrm{~Hz}), 130.5,129.4,128.3,127.9$ (d, $J=9.9 \mathrm{~Hz}), 126.3,125.5,125.1$, 123.9, 123.0, 119.7 (d, $J=25.4 \mathrm{~Hz}), 118.1,110.6(\mathrm{~d}, J=21.7 \mathrm{~Hz})$; HRMS (ESI): calculated for $\mathrm{C}_{24} \mathrm{H}_{14} \mathrm{FNO}_{2}[\mathrm{M}+\mathrm{H}]^{+}: 368.1081$, found 368.1085 .

3-(6-Bromoquinolin-2-yl)-2-phenyl-4H-chromen-4-one (12)

Brown solid, $74 \%$ yield; $\mathrm{mp}=176.3-177.3{ }^{\circ} \mathrm{C} ;{ }^{1} \mathrm{H}$ NMR $\left(600 \mathrm{MHz}, \mathrm{CDCl}_{3}\right): \delta=8.31-8.30(\mathrm{~m}, 1$ H ), 8.06-8.04 (m, 1 H), 7.97 (m, 1 H), 7.80-7.78 (m, 1 H), 7.74-7.69 (m, 2 H), 7.58-7.51 (m, 2 H), 7.46-7.38 (m, $3 \mathrm{H}), 7.31-7.28$ (m, $1 \mathrm{H}), 7.21-7.19(\mathrm{~m}, 2 \mathrm{H}) ;{ }^{13} \mathrm{C} \mathrm{NMR}\left(150 \mathrm{MHz}, \mathrm{CDCl}_{3}\right): \delta=177.2$, 163.6, 156.2, 154.3, 146.7, 135.1, 134.1, 132.9, 132.8, 131.2, 130.5, 129.6, 129.3, 128.29, 128.25, 126.3, 125.5, 125.2, 123.8, 122.9, 120.7, 118.1; HRMS (ESI): calculated for $\mathrm{C}_{24} \mathrm{H}_{14} \mathrm{NO}_{2} \mathrm{Br}[\mathrm{M}+\mathrm{H}]^{+}$: 428.0281, found 428.0283 .

3-(6-Methoxyquinolin-2-yl)-2-phenyl-4H-chromen-4-one (13)

Brown solid, $82 \%$ yield; $\mathrm{mp}=198.1-199.6{ }^{\circ} \mathrm{C} ;{ }^{1} \mathrm{H}$ NMR $\left(600 \mathrm{MHz}, \mathrm{CDCl}_{3}\right): \delta=8.31-8.30(\mathrm{~m}, 1$ H ), 8.05-8.03 (m, $1 \mathrm{H}), 7.84$-7.83 (m, $1 \mathrm{H})$, 7.73-7.70 (m, $1 \mathrm{H})$, 7.57-7.56 (m, $1 \mathrm{H})$, 7.45-7.40 (m, 4 H), 7.31-7.27 (m, 2 H), 7.21-7.18 (m, 2 H), 7.07-7.06 (m, 1 H), 3.91 (s, $3 \mathrm{H}) ;{ }^{13} \mathrm{C} \mathrm{NMR}(150 \mathrm{MHz}$, $\left.\mathrm{CDCl}_{3}\right): \delta=177.6,163.3,158.1,156.2,151.1,144.4,135.0,134.0,133.0,131.0,130.3,129.4,128.3$, $128.2,126.4,125.3,124.5,123.9,123.2,122.2,118.1,105.1,55.6$; HRMS (ESI): calculated for $\mathrm{C}_{25} \mathrm{H}_{17} \mathrm{NO}_{3}[\mathrm{M}+\mathrm{H}]^{+}:$380.1281, found 380.1297 .

\section{2-Phenyl-3-(6-(trifluoromethoxy)quinolin-2-yl)-4H-chromen-4-one (14)}

Brown solid, $65 \%$ yield; $\mathrm{mp}=66.9-68.6{ }^{\circ} \mathrm{C} ;{ }^{1} \mathrm{H}$ NMR $(400 \mathrm{MHz}$, Chloroform- $d) \delta 8.8(\mathrm{~d}, J=9.4$ $\mathrm{Hz}, 2 \mathrm{H}), 8.5(\mathrm{~d}, J=5.7 \mathrm{~Hz}, 2 \mathrm{H}), 7.8-7.7(\mathrm{~m}, 4 \mathrm{H}), 7.6(\mathrm{~d}, J=9.3 \mathrm{~Hz}, 2 \mathrm{H}), 7.4(\mathrm{t}, J=7.0 \mathrm{~Hz}, 2 \mathrm{H})$, $7.3-7.3(\mathrm{~m}, 1 \mathrm{H}) .{ }^{13} \mathrm{C}$ NMR $(101 \mathrm{MHz}$, Chloroform- $d$ ) $\delta$ 177.2, 163.6, 154.3, 147.1, 146.2, 134.1, 132.7, $131.7,130.5,129.3,128.2,127.3,126.3,125.4,125.3,123.7,123.5,122.7,121.8,119.2(\mathrm{q}, J=257.9$ $\mathrm{Hz}$ ), 118.1, 117.5. HRMS (ESI): calculated for $\mathrm{C}_{25} \mathrm{H}_{15} \mathrm{~F}_{3} \mathrm{NO}_{3}[\mathrm{M}+\mathrm{H}]^{+}: 434.0998$, found 434.0998.

3-(7-Methoxyquinolin-2-yl)-2-phenyl-4H-chromen-4-one (15)

Brown solid, $72 \%$ yield; $\mathrm{mp}=140.0-141.2{ }^{\circ} \mathrm{C} ;{ }^{1} \mathrm{H}$ NMR $\left(600 \mathrm{MHz}, \mathrm{CDCl}_{3}\right): \delta=8.31-8.30(\mathrm{~m}, 1$ 
H ), 8.05-8.03 (m, $1 \mathrm{H}), 7.72-7.66(\mathrm{~m}, 2 \mathrm{H}), 7.57-7.55(\mathrm{~m}, 1 \mathrm{H}), 7.44-7.42(\mathrm{~m}, 3 \mathrm{H}), 7.32-7.27(\mathrm{~m}$, $3 \mathrm{H}), 7.21-7.17$ (m, $3 \mathrm{H}) 3.87$ (s, $3 \mathrm{H}) ;{ }^{13} \mathrm{C}$ NMR $\left(150 \mathrm{MHz}, \mathrm{CDCl}_{3}\right): \delta=177.4,162.9,160.6,156.1$, $153.9,149.9,135.9,133.9,132.9,130.4,129.3,128.6,128.2,126.3,125.3,123.8,123.3,122.4$, 122.0, 120.0, 118.0, 107.5, 55.5; HRMS (ESI): calculated for $\mathrm{C}_{25} \mathrm{H}_{17} \mathrm{NO}_{3}[\mathrm{M}+\mathrm{H}]^{+}: 380.1281$, found 380.1295 .

3-(8-Methoxyquinolin-2-yl)-2-phenyl-4H-chromen-4-one (16)

Brown solid, $52 \%$ yield; $\mathrm{mp}=154.3-155.6{ }^{\circ} \mathrm{C} ;{ }^{1} \mathrm{H}$ NMR $\left(600 \mathrm{MHz}, \mathrm{CDCl}_{3}\right): \delta=8.30-8.28(\mathrm{~m}, 1$ H ), 8.10-8.08 (m, $1 \mathrm{H}), 7.71-7.68(\mathrm{~m}, 1 \mathrm{H}), 7.55-7.53(\mathrm{~m}, 1 \mathrm{H}), 7.48-7.40(\mathrm{~m}, 5 \mathrm{H}), 7.37-7.35(\mathrm{~m}$, $1 \mathrm{H}), 7.28-7.25$ (m, $1 \mathrm{H}), 7.20-7.17$ (m, $2 \mathrm{H}), 7.00-6.98$ (m, $1 \mathrm{H}), 3.92-3.90(\mathrm{~m}, 3 \mathrm{H}) ;{ }^{13} \mathrm{C} \mathrm{NMR}$ $\left(150 \mathrm{MHz}, \mathrm{CDCl}_{3}\right): \delta=177.6,163.2,156.1,155.8,152.4,140.3,136.1,133.9,133.1,130.2,129.5$, 128.4, 128.1, 126.9, 126.3, 125.3, 124.7, 123.9, 123.6, 119.5, 118.0, 108.2, 56.2; HRMS (ESI): calculated for $\mathrm{C}_{24} \mathrm{H}_{17} \mathrm{~N}_{2} \mathrm{O}[\mathrm{M}+\mathrm{H}]^{+}: 380.1281$, found 380.1296 .

3-(3-Methylquinolin-2-yl)-2-phenyl-4H-chromen-4-one (17)

Brown solid, 77\% yield; $\mathrm{mp}=185.6-186.2{ }^{\circ} \mathrm{C} ;{ }^{1} \mathrm{H}$ NMR $\left(400 \mathrm{MHz}, \mathrm{CD}_{2} \mathrm{Cl}_{2}\right): \delta=8.22-8.20(\mathrm{~m}, 1$ H ), 7.91-7.89 (m, 1 H), 7.76 -7.73 (m, 2 H), 7.61-7.59 (m, 1 H), 7.52-7.43 (m, 6 H), 7.31-7.28 (m, $1 \mathrm{H}), 7.23-7.19$ (m, $2 \mathrm{H}), 3.76$ (s, $3 \mathrm{H}) ;{ }^{13} \mathrm{C} \mathrm{NMR}\left(150 \mathrm{MHz}, \mathrm{CDCl}_{3}\right): \delta=177.3,162.9,156.4,152.6$, $148.0,143.3,133.9,133.2,130.4,129.5,129.3,128.8,128.3,127.2,126.8,126.5,126.4,125.3$, 123.8, 120.6, 118.1, 112.8, 55.8; HRMS (ESI): calculated for $\mathrm{C}_{25} \mathrm{H}_{17} \mathrm{NO}_{3}[\mathrm{M}+\mathrm{Na}]^{+}: 402,1101$, found 402.1112 .

2-(4-Chlorophenyl)-3-(6-methoxyquinolin-2-yl)-4H-chromen-4-one (18)

Brown solid, 79\% yield; mp $=222.7-224.1{ }^{\circ} \mathrm{C} .{ }^{1} \mathrm{H}$ NMR $\left(600 \mathrm{MHz}, \mathrm{CDCl}_{3}\right): \delta=8.30-8.28(\mathrm{~m}, 1$ H ), 8.07-8.06 (m, $1 \mathrm{H})$, 7.84-7.83 (m, $1 \mathrm{H}), 7.73-7.70$ (m, $1 \mathrm{H})$, 7.56-7.54 (m, $1 \mathrm{H})$, 7.46-7.43 (m, $2 \mathrm{H})$, 7.34-7.31 (m, $3 \mathrm{H}), 7.17-7.16$ (m, $2 \mathrm{H}), 7.08-7.079$ (m, $1 \mathrm{H}), 3.92$ (s, $3 \mathrm{H}) ;{ }^{13} \mathrm{C}$ NMR (150 $\left.\mathrm{MHz}, \mathrm{CDCl}_{3}\right): \delta=177.4,162.0,158.2,156.1,150.7,144.4,136.5,135.2,134.1,131.5,131.0,130.7$, 128.5, 128.4, 126.4, 125.5, 124.4, 123.8, 123.3, 122.4, 118.1, 105.1, 55.7; HRMS (ESI): calculated for $\mathrm{C}_{25} \mathrm{H}_{16} \mathrm{ClNO}_{3}[\mathrm{M}+\mathrm{Na}]^{+}$: 436.0711, found 436.0715. 
3-(6-Methoxyquinolin-2-yl)-2-(4-(trifluoromethyl)phenyl)-4H-chromen-4-one (19)

Brown solid, $71 \%$ yield; $\mathrm{mp}=204.1-206.0{ }^{\circ} \mathrm{C} ;{ }^{1} \mathrm{H}$ NMR $\left(600 \mathrm{MHz}, \mathrm{CDCl}_{3}\right): \delta=8.17-8.16(\mathrm{~m}, 1$ H ), 7.93-7.92 (m, $1 \mathrm{H})$, 7.69-7.67 (m, $1 \mathrm{H})$, 7.58-7.55 (m, $1 \mathrm{H})$, 7.42-7.39 (m, $3 \mathrm{H})$, 7.36-7.28 (m, $4 \mathrm{H}), 7.18-7.16(\mathrm{~m}, 1 \mathrm{H}), 6.94-6.93(\mathrm{~m}, 1 \mathrm{H}), 3.75(\mathrm{~s}, 3 \mathrm{H}) ;{ }^{13} \mathrm{C} \mathrm{NMR}\left(150 \mathrm{MHz}, \mathrm{CDCl}_{3}\right): \delta=177.2$, $161.4,158.2,156.0,150.2,144.2,136.6,135.1,134.1,131.7$ (q, $J=32.3 \mathrm{~Hz}), 130.8,129.7,128.3$, $126.2,125.5,125.0(\mathrm{q}, J=3.5 \mathrm{~Hz}), 124.3,123.8,123.7,123.6(\mathrm{q}, J=271.1 \mathrm{~Hz}), 122.4,118.0,105.0$ 55.5. HRMS (ESI): calculated for $\mathrm{C}_{26} \mathrm{H}_{16} \mathrm{~F}_{3} \mathrm{NO}_{3}[\mathrm{M}+\mathrm{H}]^{+}: 448.1155$, found 448.1157 .

2-(4-Methoxyphenyl)-3-(6-methoxyquinolin-2-yl)-4H-chromen-4-one (20)

Brown solid, $65 \%$ yield; $\mathrm{mp}=202.3-203.4{ }^{\circ} \mathrm{C} ;{ }^{1} \mathrm{H}$ NMR $\left(600 \mathrm{MHz}, \mathrm{CDCl}_{3}\right): \delta=8.29-8.28(\mathrm{~m}, 1$ H ), 8.06-8.05 (m, $1 \mathrm{H})$, 7.90-7.89 (m, $1 \mathrm{H}), 7.71-7.68(\mathrm{~m}, 1 \mathrm{H})$, 7.56-7.54 (m, $1 \mathrm{H})$, 7.43-7.41 (m, $2 \mathrm{H}), 7.35-7.31(\mathrm{~m}, 3 \mathrm{H}), 7.084(\mathrm{~m}, 1 \mathrm{H}), 6.70-6.69(\mathrm{~m}, 2 \mathrm{H}), 3.93(\mathrm{~s}, 3 \mathrm{H}), 3.72(\mathrm{~s}, 3 \mathrm{H}) ;{ }^{13} \mathrm{C}$ NMR $\left(150 \mathrm{MHz}, \mathrm{CDCl}_{3}\right): \delta=177.6,163.0,161.2,158.1,156.2,151.5,144.5,135.2,133.8,131.14,131.12$, $128.3,126.3,125.2,124.6,123.9,122.3,122.1,118.0,113.7,105.1,55.6,55.3$; HRMS (ESI): calculated for $\mathrm{C}_{26} \mathrm{H}_{19} \mathrm{NO}_{4}[\mathrm{M}+\mathrm{H}]^{+}: 410.1387$, found 410.1393 .

\section{3-(6-Methoxyquinolin-2-yl)-2-(p-tolyl)-4H-chromen-4-one (21)}

Brown solid, $79 \%$ yield; $\mathrm{mp}=171.1-174.1{ }^{\circ} \mathrm{C} ;{ }^{1} \mathrm{H}$ NMR $\left(600 \mathrm{MHz}, \mathrm{CDCl}_{3}\right): \delta=8.30-8.29(\mathrm{~m}, 1$ H ), 8.05-8.03 (m, $1 \mathrm{H})$, 7.89-7.87 (m, $1 \mathrm{H})$, 7.72-7.69 (m, $1 \mathrm{H})$, 7.56-7.55 (m, $1 \mathrm{H})$, 7.44-7.41 (m, $2 \mathrm{H}), 7.32-7.29$ (m, $3 \mathrm{H}), 7.079-7.075$ (m, $1 \mathrm{H}), 7.00-6.99$ (m, $2 \mathrm{H}), 3.93$ (s, $3 \mathrm{H}), 2.26(\mathrm{~s}, 3 \mathrm{H}) ;{ }^{13} \mathrm{C}$ $\operatorname{NMR}\left(150 \mathrm{MHz}, \mathrm{CDCl}_{3}\right): \delta=177.6,163.3,158.1,156.2,151.4,144.5,140.8,135.1,133.9,131.1$, 130.2, 129.4, 129.0, 128.3, 126.4, 125.3, 124.6, 123.9, 122.9, 122.1, 118.1, 105.1, 55.7, 21.5; HRMS (ESI): calculated for $\mathrm{C}_{26} \mathrm{H}_{19} \mathrm{NO}_{3}[\mathrm{M}+\mathrm{H}]^{+}$: 394.1438 , found 394.1442 .

2-(4-Fluorophenyl)-3-(6-methoxyquinolin-2-yl)-4H-chromen-4-one (22)

Brown solid, $80 \%$ yield; $\mathrm{mp}=200.2-202.8{ }^{\circ} \mathrm{C} ;{ }^{1} \mathrm{H}$ NMR $\left(600 \mathrm{MHz}, \mathrm{CDCl}_{3}\right): \delta=8.31-8.29(\mathrm{~m}, 1$ H ), 8.08-8.06 (m, $1 \mathrm{H})$, 7.84-7.83 (m, $1 \mathrm{H})$, 7.74-7.72 (m, $1 \mathrm{H})$, 7.57-7.56 (m, $1 \mathrm{H})$, 7.46-7.44 (m, 2 H), 7.42-7.39 (m, 2 H), 7.33-7.31 (m, 1 H), 7.090-7.086 (m, 1 H), 6.90-6.88 (m, 2 H), 3.94 (s, 3 $\mathrm{H}) ;{ }^{13} \mathrm{C}$ NMR $\left(150 \mathrm{MHz}, \mathrm{CDCl}_{3}\right): \delta=177.4,163.5,162.1,158.1,156.0,150.8,144.3,135.1,134.0$, 
$131.5,130.8,129.1,128.3,126.2,125.3,124.4,123.7,123.0,122.3,118.0,115.3,105.0,55.5$;

HRMS (ESI): calculated for $\mathrm{C}_{25} \mathrm{H}_{16} \mathrm{FNO}_{3}[\mathrm{M}+\mathrm{H}]^{+}: 398.1187$, found 398.1189 .

\section{References}

1. Yoshida, M.; Fujino, Y.; Doi, T., Synthesis of $\gamma$-benzopyranone by TfOH-promoted regioselective cyclization of o-alkynoylphenols. Organic letters 2011, 13 (17), 4526-4529.

2. Changqing; Liu; Zhannan; Zhang; Jitan; Zhang; Xing; Liu; Meihua; Xie, Regioselective synthesis of aurone derivatives via $\mathrm{PBu}_{3}$-catalyzed cyclization of 2-alkynoylphenols. Chinese Journal of Chemistry 2014, 32(12), 1233-1237.

3. Yoshida, M.; Saito, K.; Fujino, Y.; Doi, T., A concise total synthesis of biologically active frutinones via tributylphosphine-catalyzed tandem acyl transfer-cyclization. Tetrahedron 2014, 70 (21), 3452-3458.

4. Saito, K.; Yoshida, M.; Doi, T., An efficient synthesis of aurone derivatives by the tributylphosphinecatalyzed regioselective cyclization of o-alkynoylphenols. Chemistry Letters 2015, 44 (2), 141-143.

5. Zhang, S.; Wu, C.; Zhang, Z.; Wang, T., Metal-free synthesis of 3-(iso) quinolinyl 4-chromenones and 3-(sso) quinolinyl 4-quinolones from (iso) quinoline N-Oxides and Ynones. Organic Letters 2019, 21 (24), 9995-9998. 


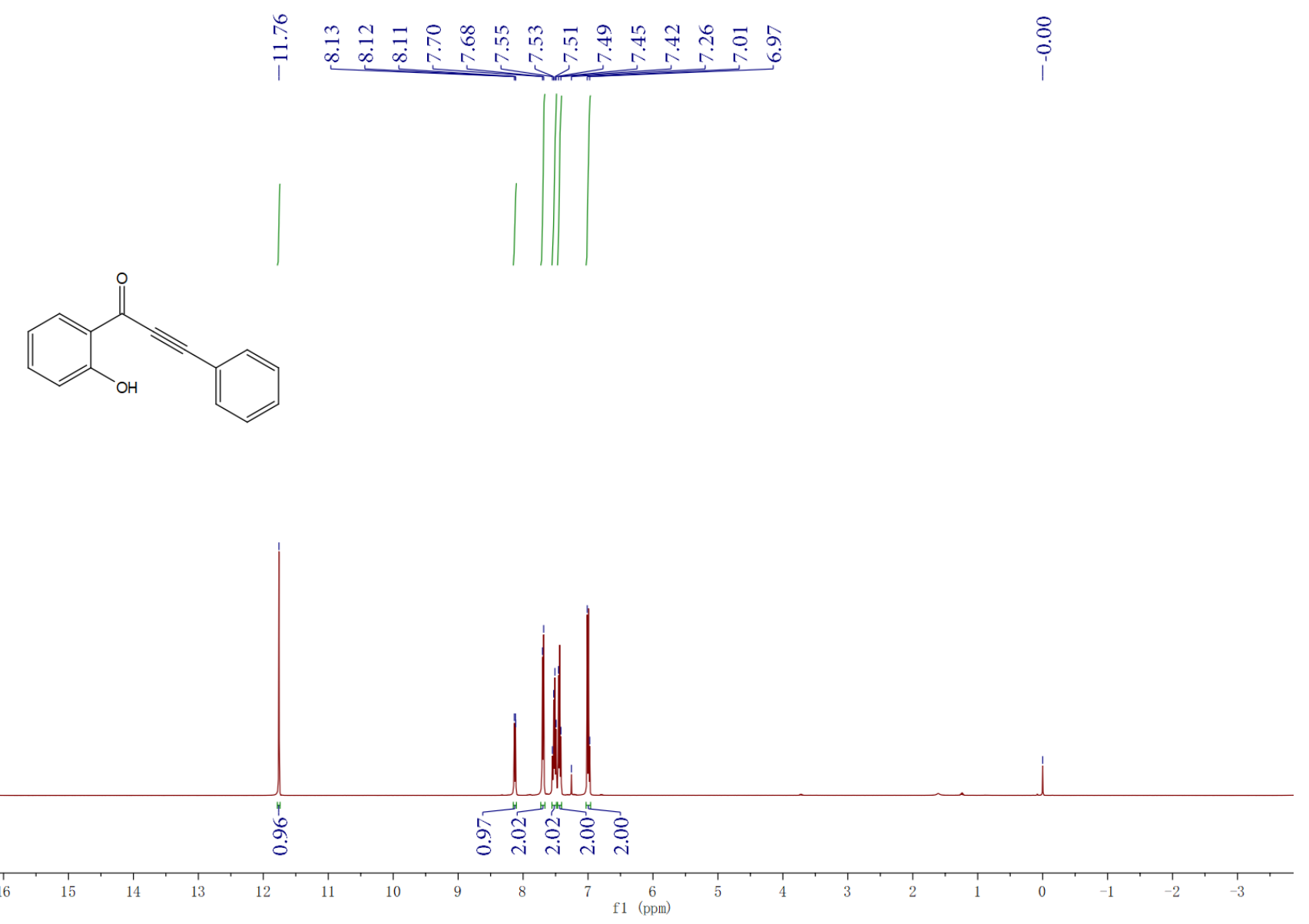

${ }^{1} \mathrm{H}$ NMR of compound $\mathbf{1 a}$ in chloroform- $d$

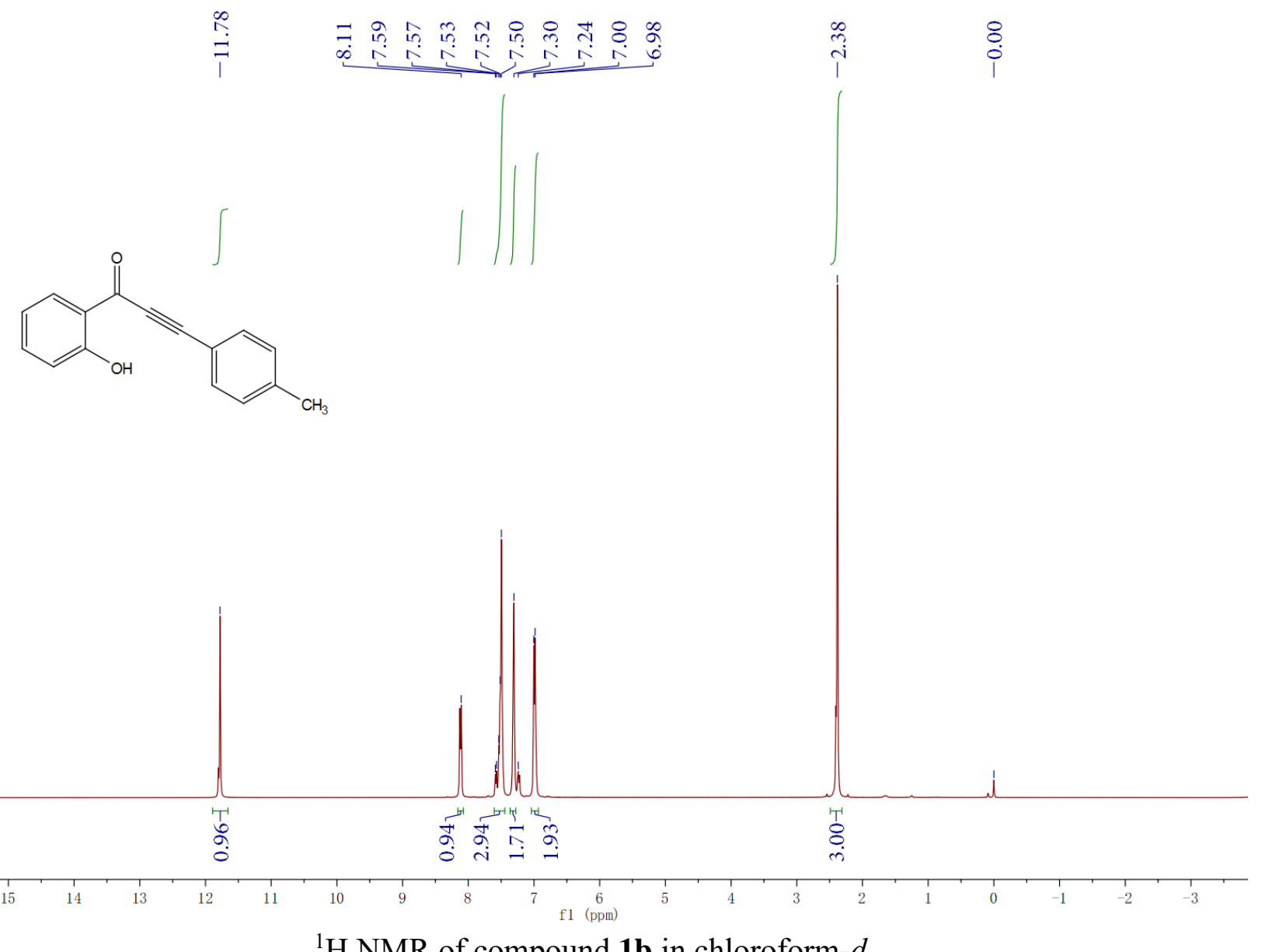

${ }^{1} \mathrm{H}$ NMR of compound $\mathbf{1 b}$ in chloroform- $d$ 

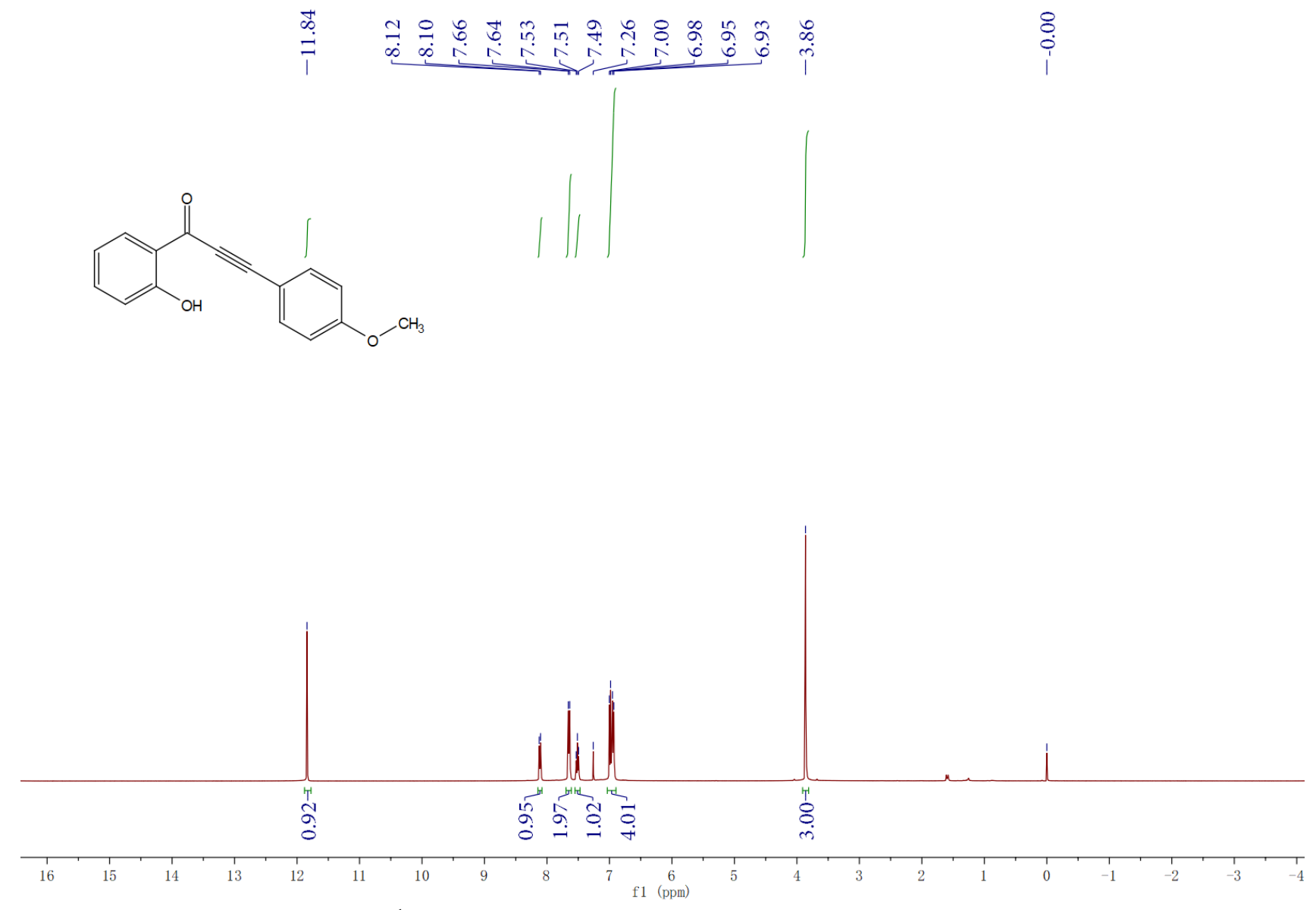

${ }^{1} \mathrm{H}$ NMR of compound 1c in chloroform- $d$
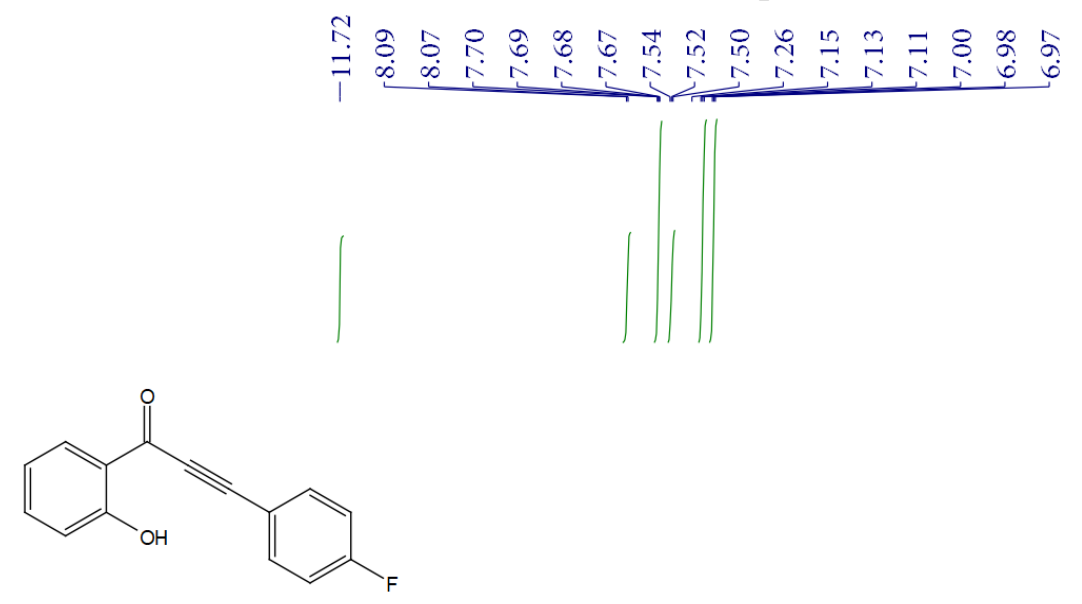

o̊
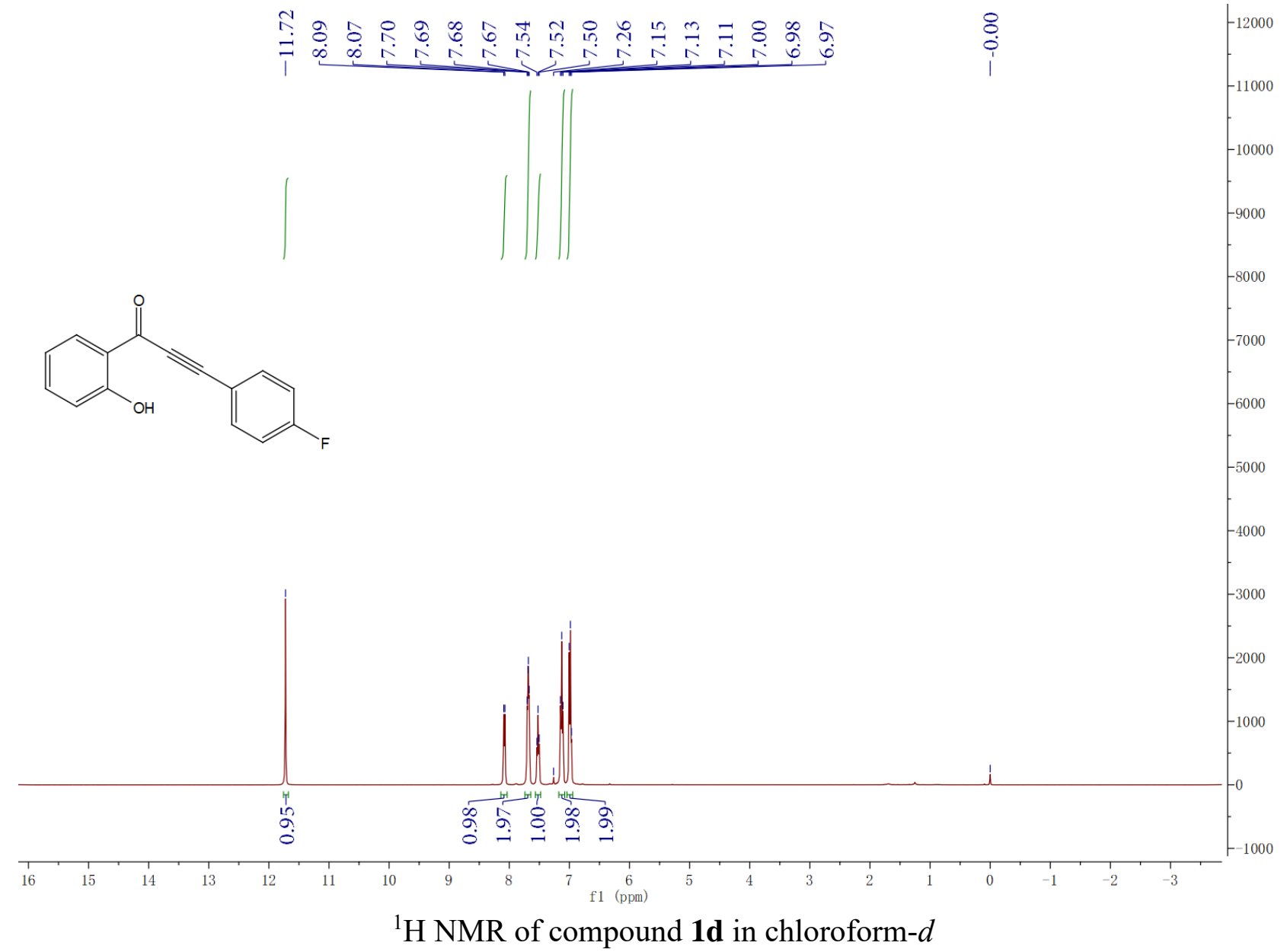

${ }^{1} \mathrm{H}$ NMR of compound $\mathbf{1 d}$ in chloroform- $d$ 

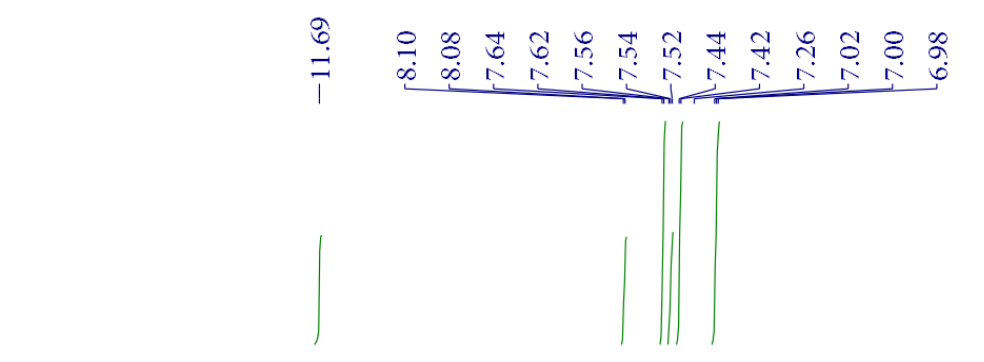<smiles>O=C(C#Cc1ccc(Cl)cc1)c1ccccc1O</smiles>

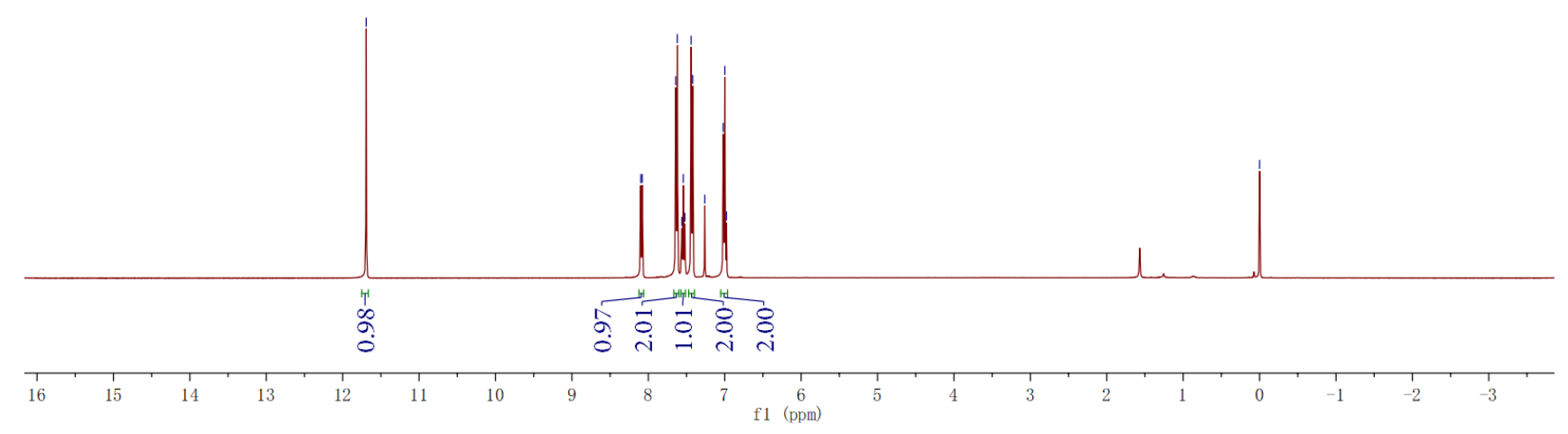

${ }^{1} \mathrm{H}$ NMR of compound $1 \mathrm{e}$ in chloroform- $d$

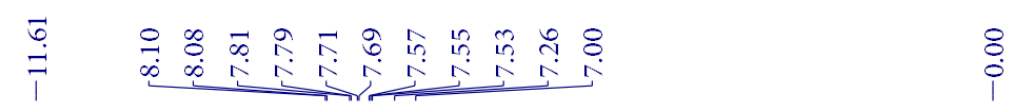
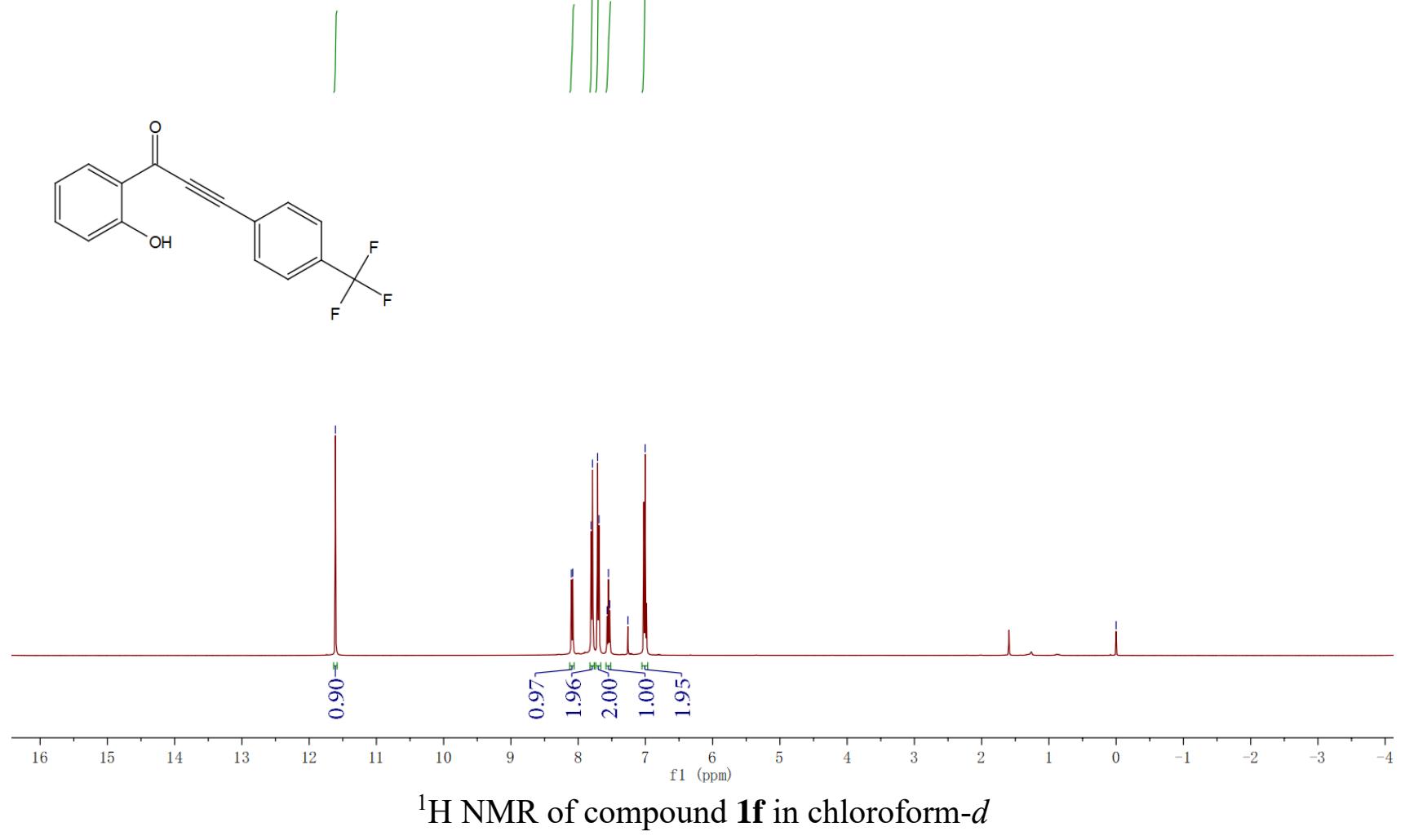


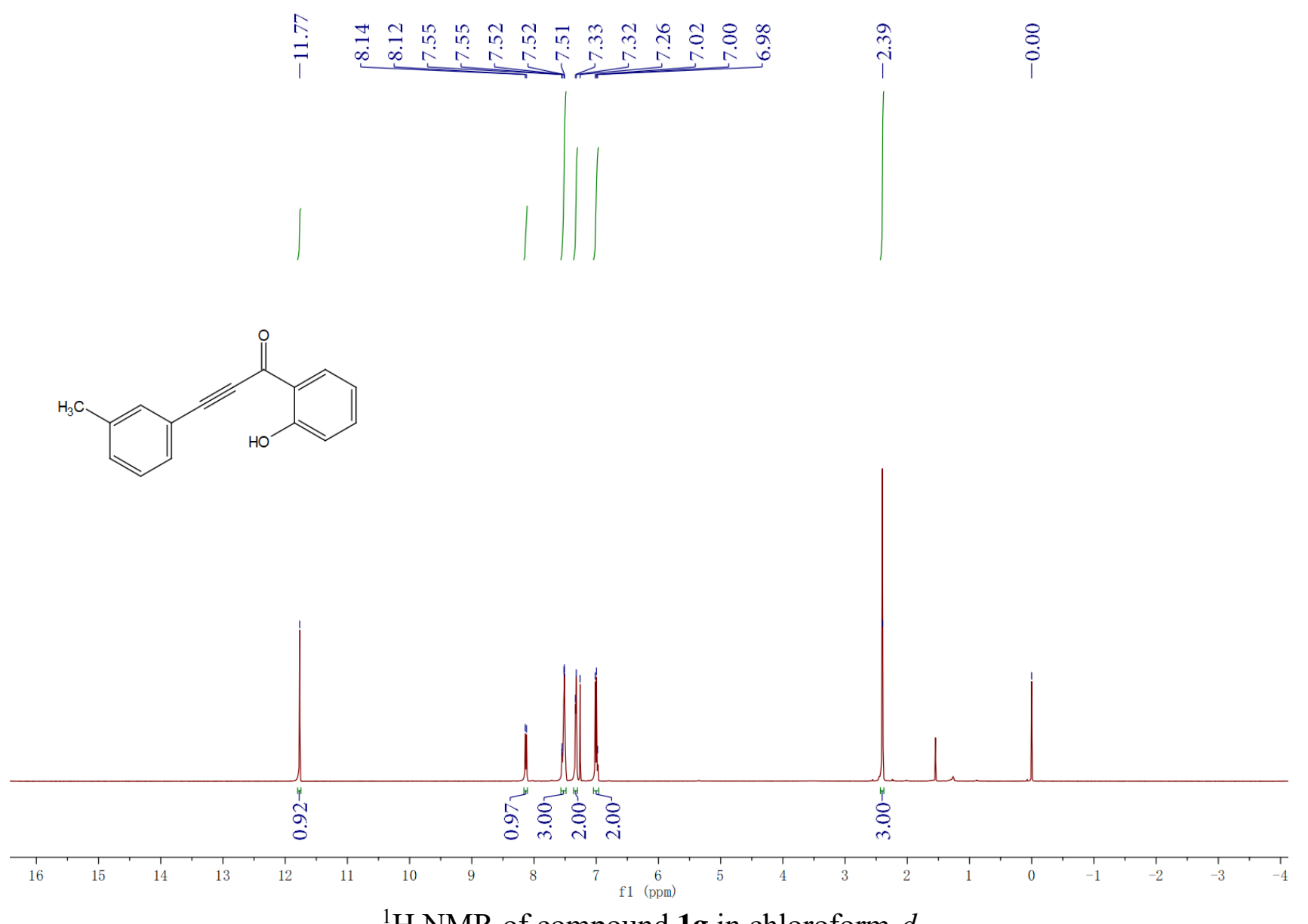

${ }^{1} \mathrm{H}$ NMR of compound $\mathbf{1 g}$ in chloroform- $d$

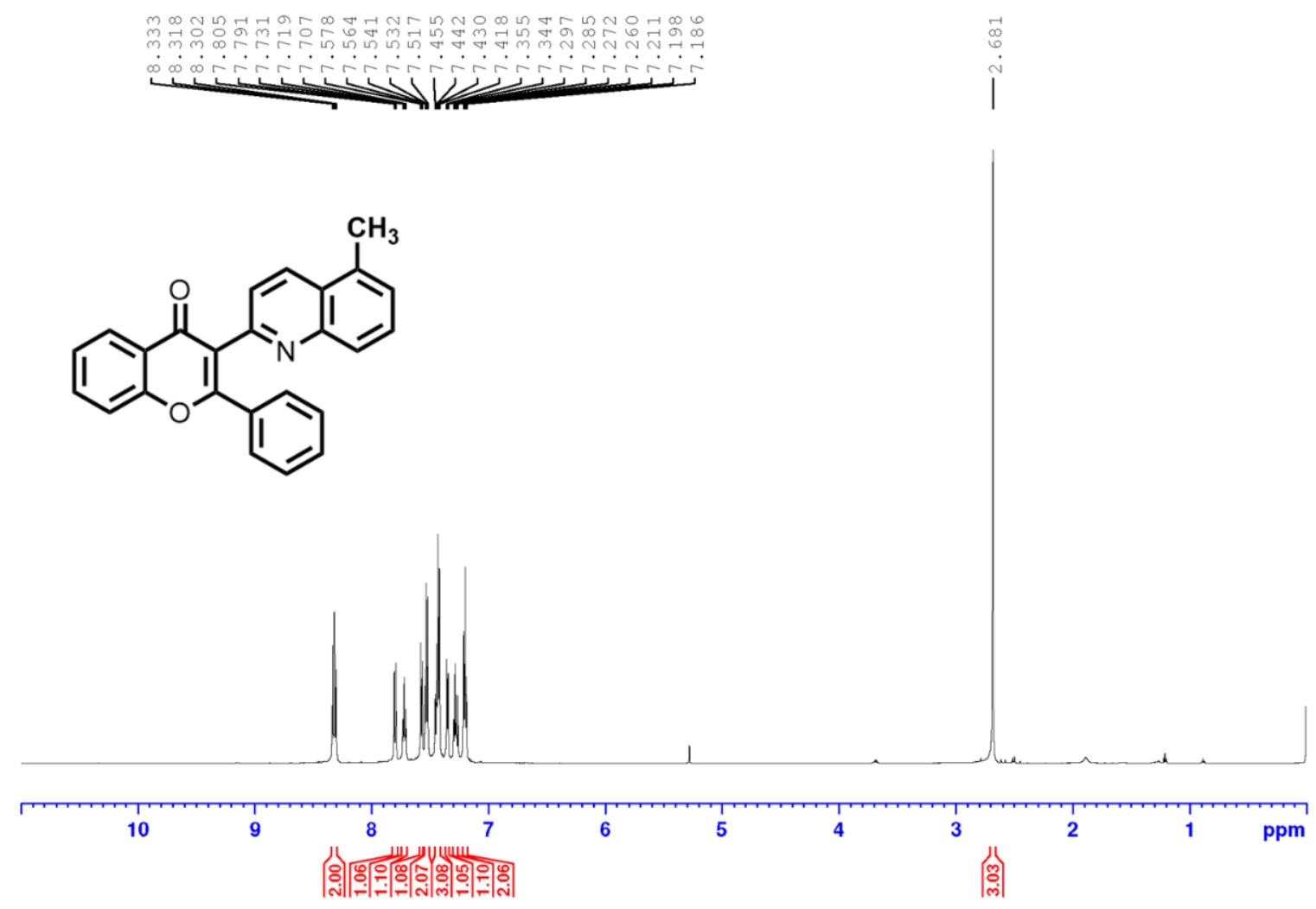

${ }^{1} \mathrm{H}$ NMR of compound $\mathbf{4}$ in chloroform- $d$ 


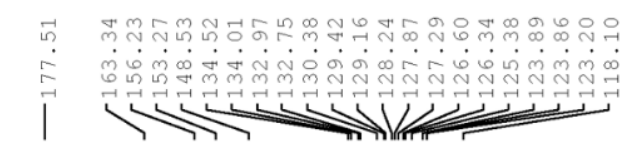<smiles>Cc1cccc2nc(-c3c(-c4ccccc4)oc4ccccc4c3=O)ccc12</smiles>
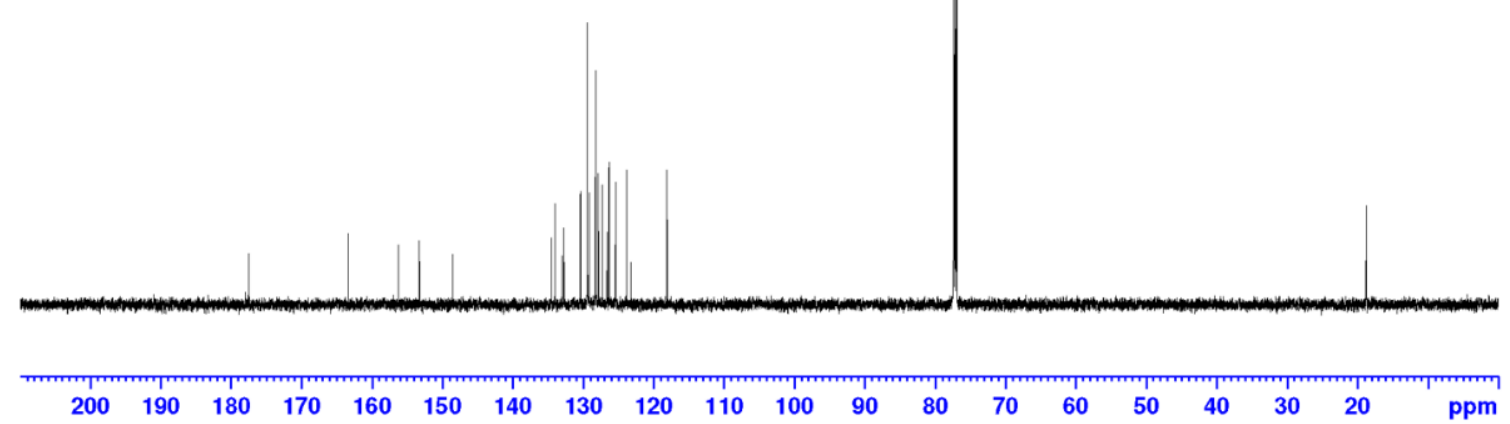

${ }^{13} \mathrm{C}$ NMR of compound 4 in chloroform- $d$

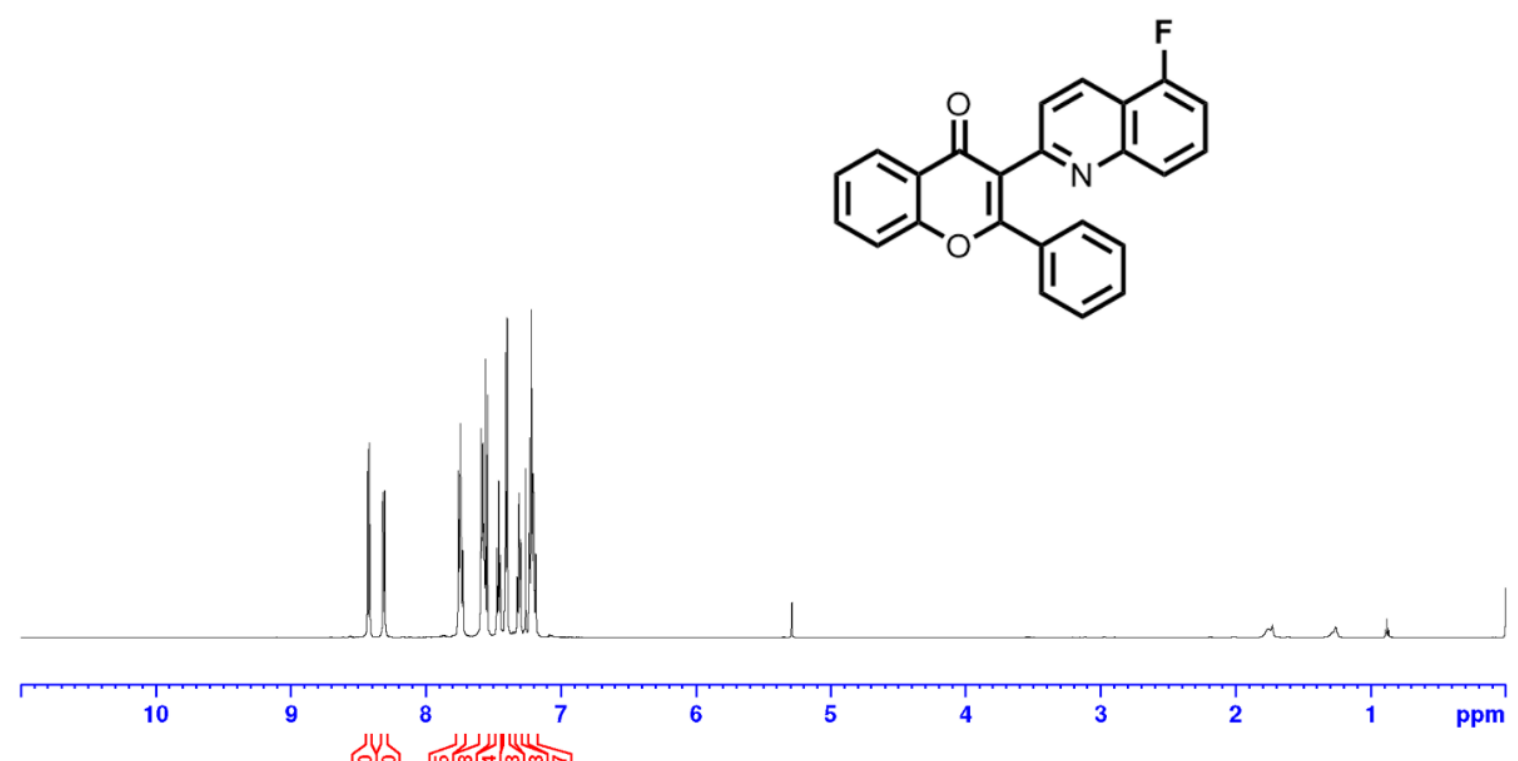

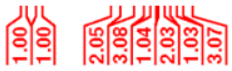

${ }^{1} \mathrm{H}$ NMR of compound $\mathbf{5}$ in chloroform- $d$ 


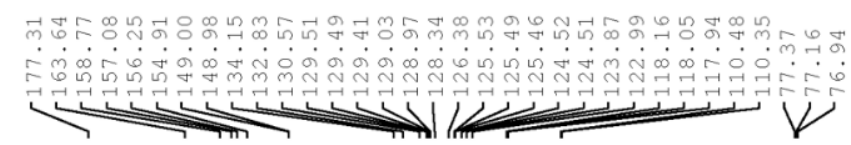<smiles>O=c1c(-c2ccc3c(F)cccc3n2)c(-c2ccccc2)oc2ccccc12</smiles>

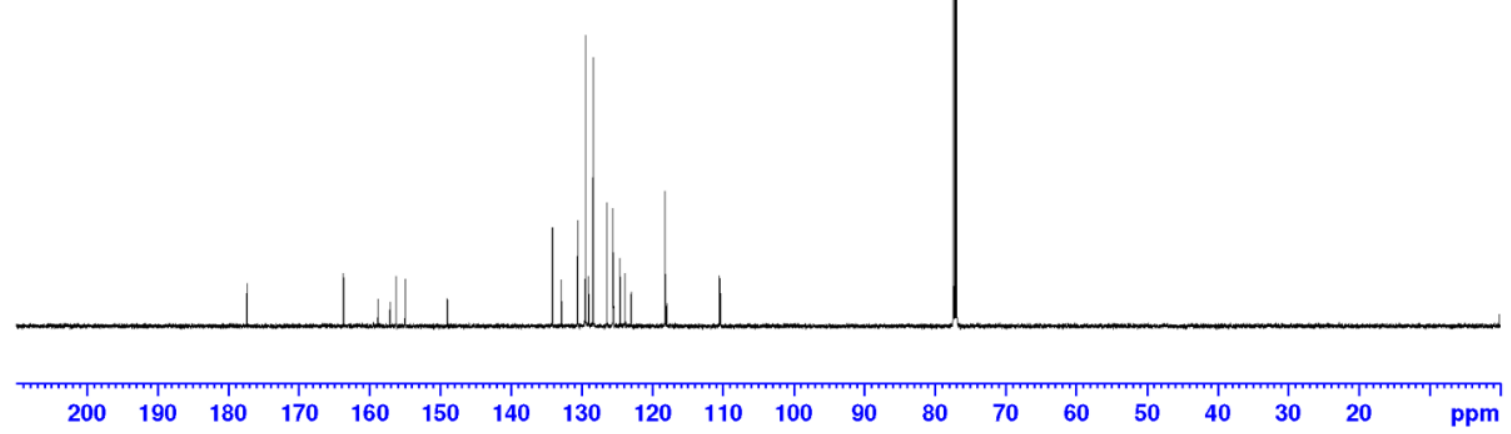

${ }^{13} \mathrm{C}$ NMR of compound 5 in chloroform- $d$<smiles>O=c1c(-c2ccc3c(Cl)cccc3n2)c(-c2ccccc2)oc2ccccc12</smiles>

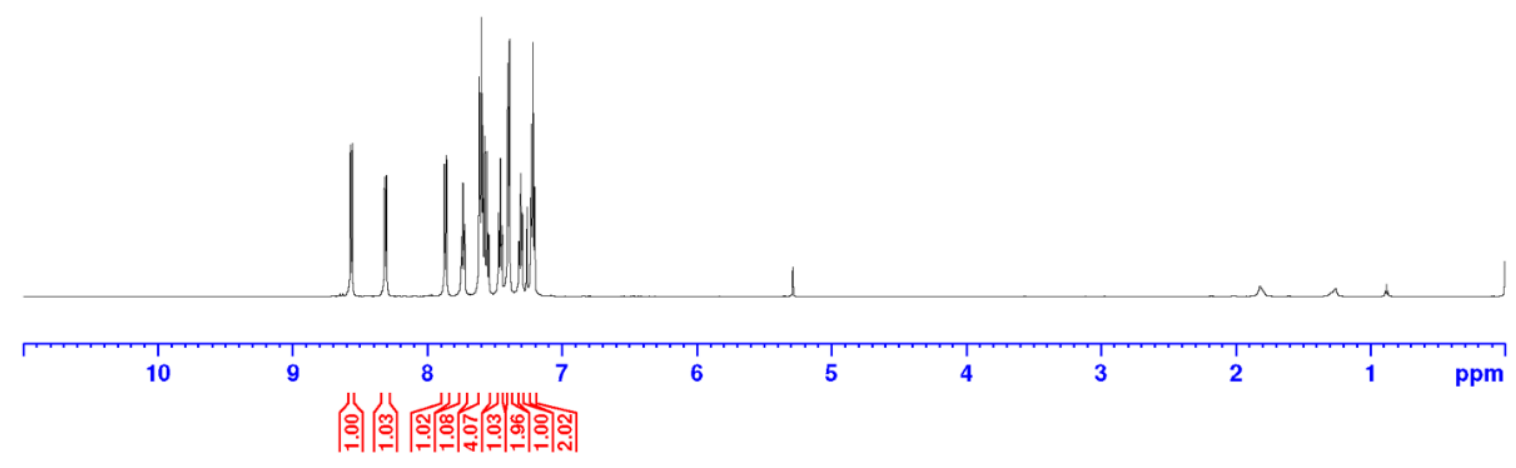

${ }^{1} \mathrm{H}$ NMR of compound $\mathbf{6}$ in chloroform- $d$ 


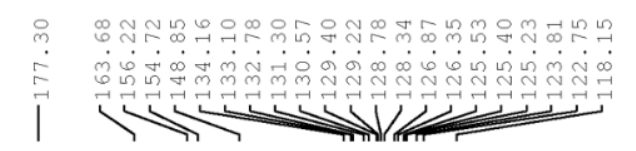<smiles>O=c1c(-c2ccc3c(Cl)cccc3n2)c(-c2ccccc2)oc2ccccc12</smiles>

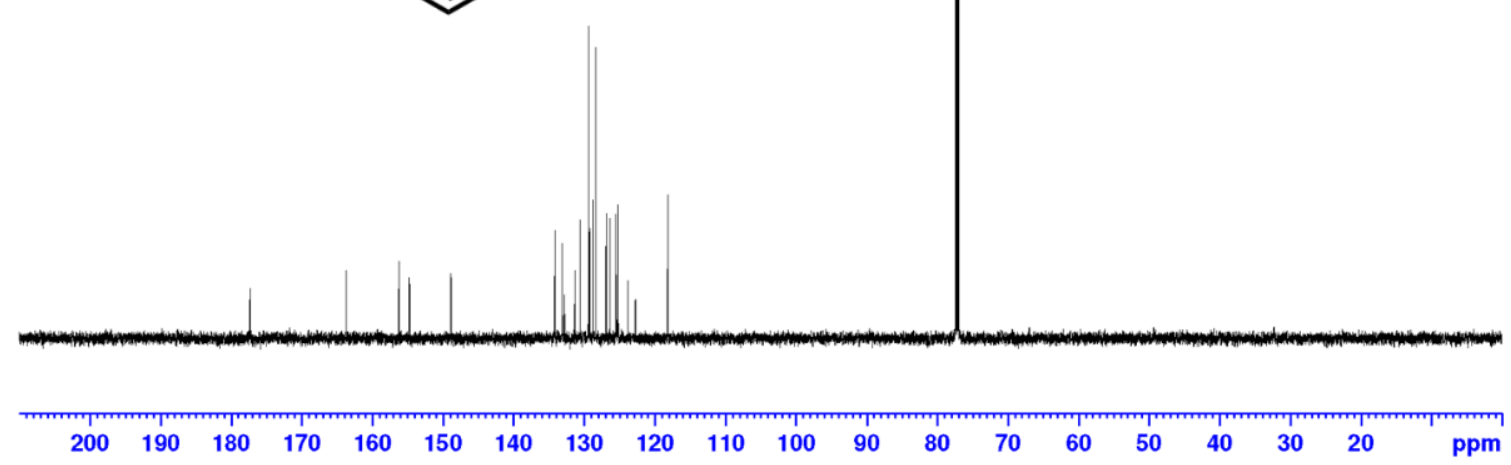

${ }^{13} \mathrm{C}$ NMR of compound $\mathbf{6}$ in chloroform- $d$<smiles>O=c1c(-c2ccc3c(Br)cccc3n2)c(-c2ccccc2)oc2ccccc12</smiles>

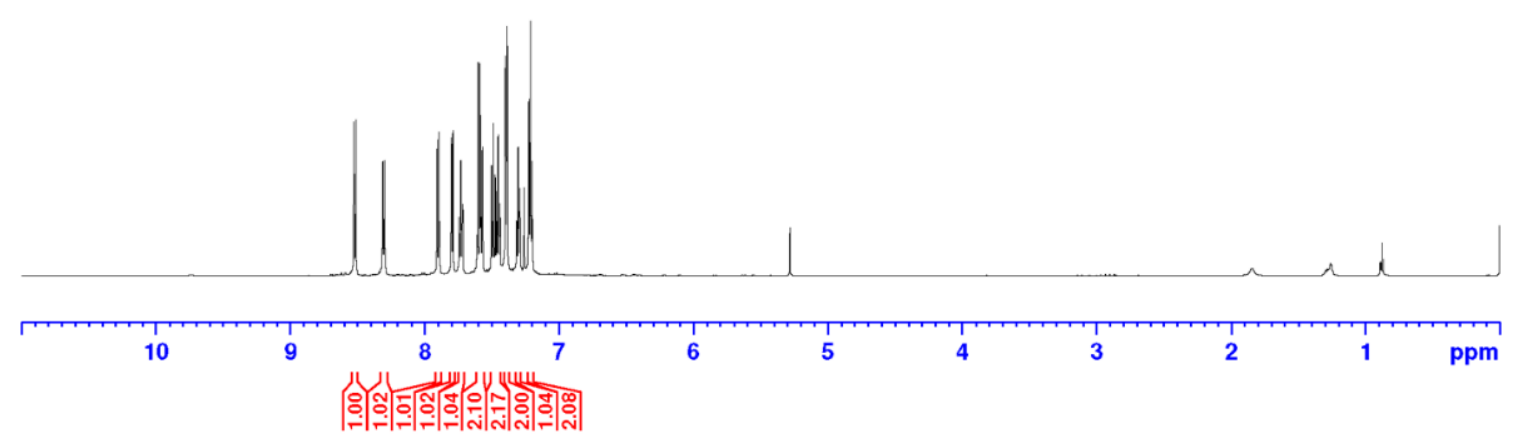

${ }^{1} \mathrm{H}$ NMR of compound $\mathbf{7}$ in chloroform- $d$ 

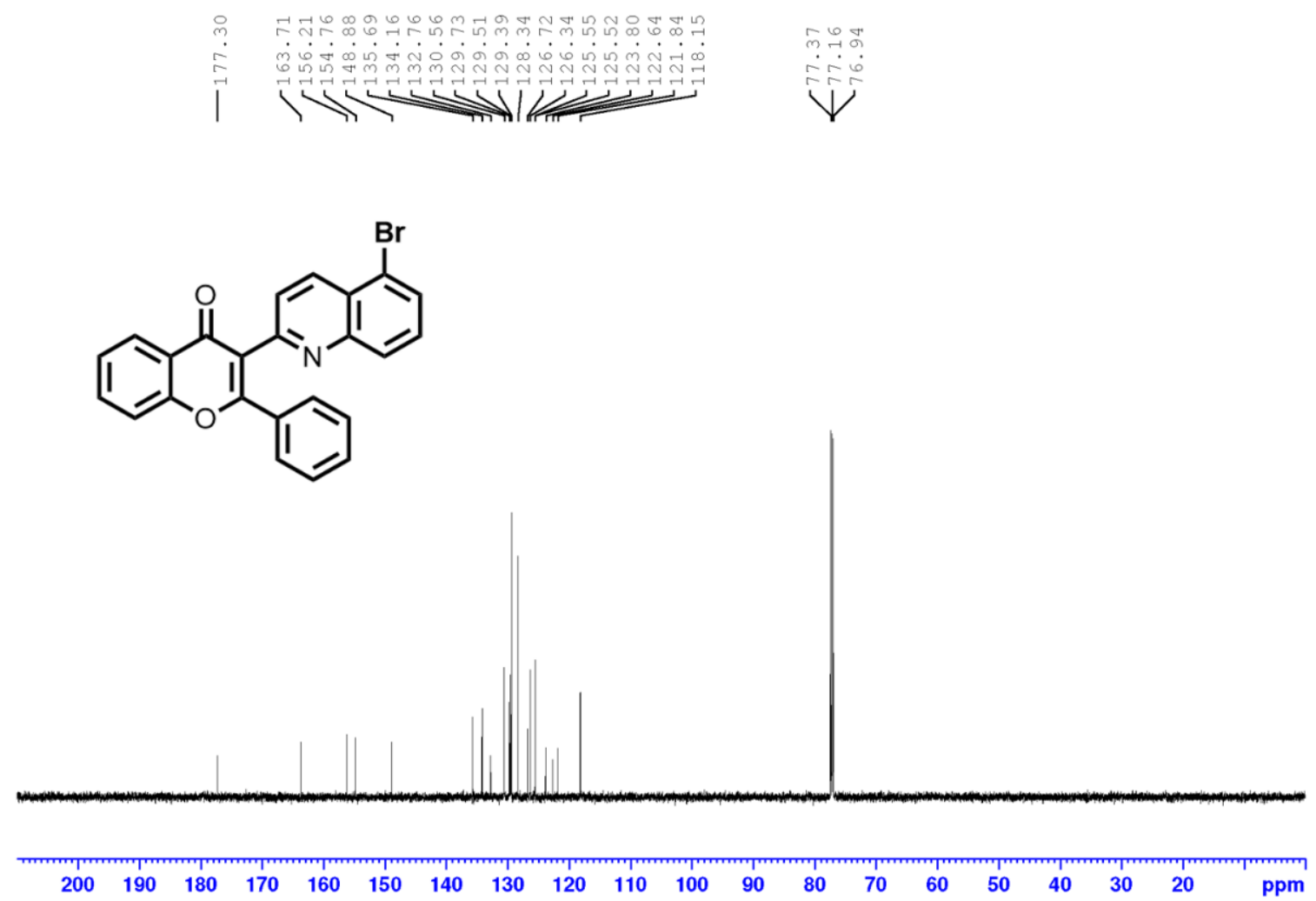

${ }^{13} \mathrm{C}$ NMR of compound 7 in chloroform- $d$
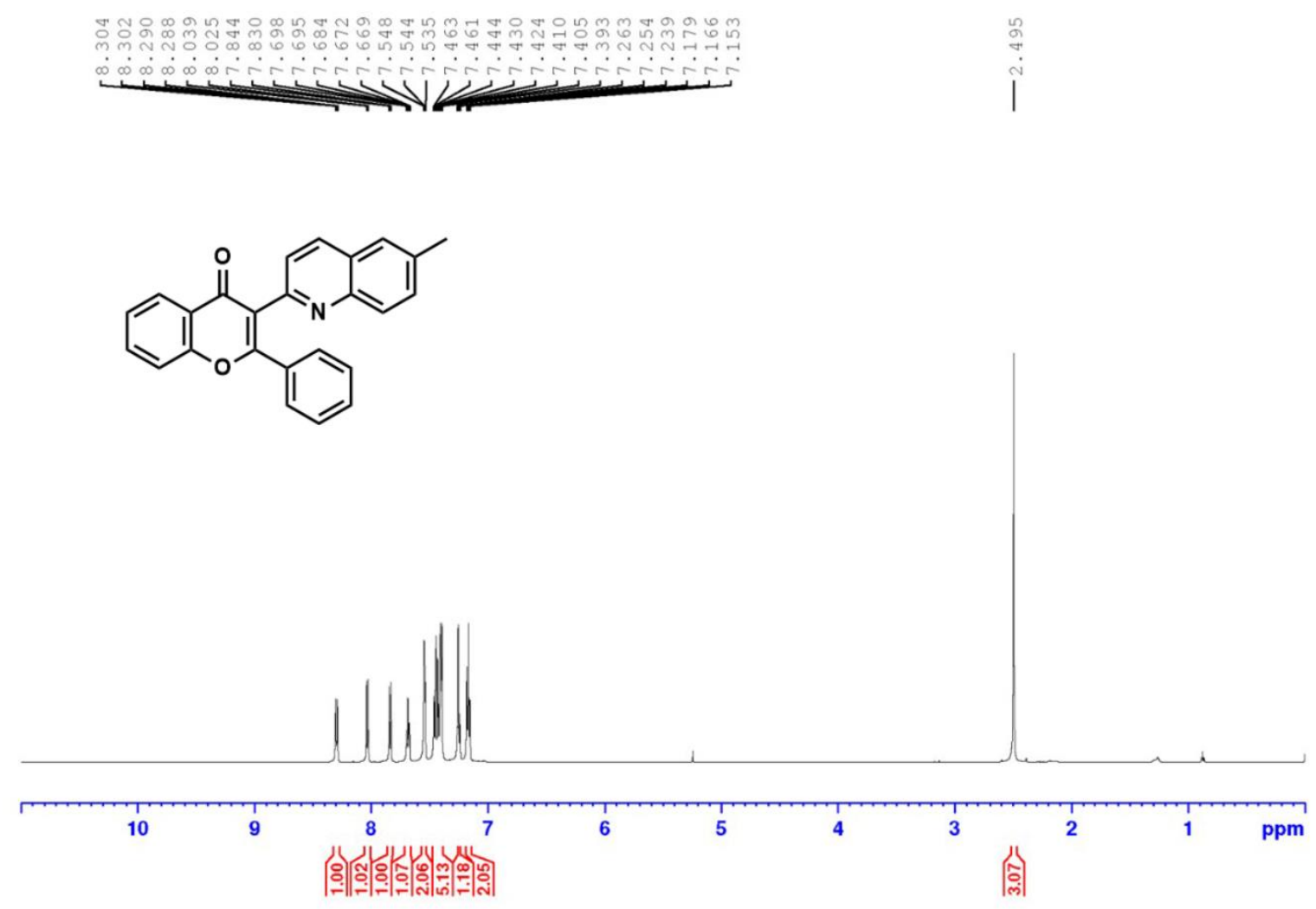

${ }^{1} \mathrm{H}$ NMR of compound 9 in chloroform- $d$ 


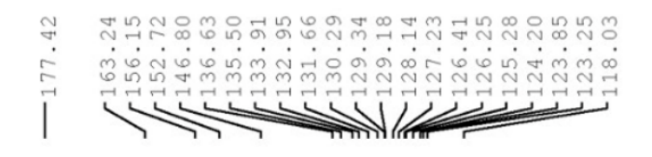

\section{踥}

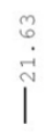<smiles>Cc1ccc2nc(-c3c(-c4ccccc4)oc4ccccc4c3=O)ccc2c1</smiles>

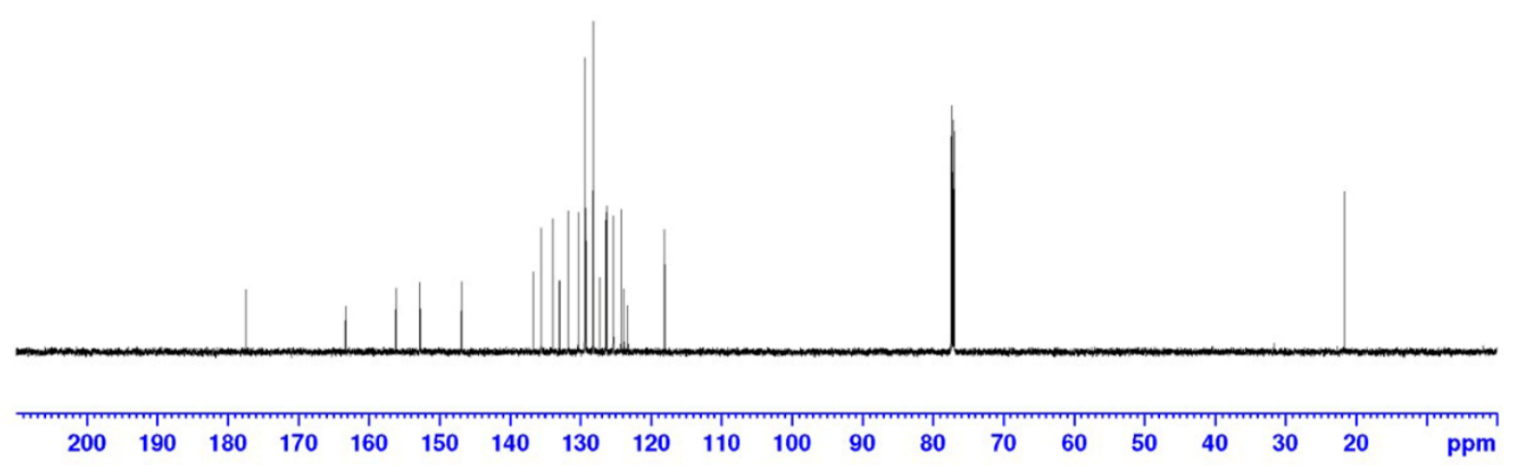

${ }^{13} \mathrm{C}$ NMR of compound 9 in chloroform- $d$<smiles>O=c1c(-c2ccc3cc(F)ccc3n2)c(-c2ccccc2)oc2ccccc12</smiles>

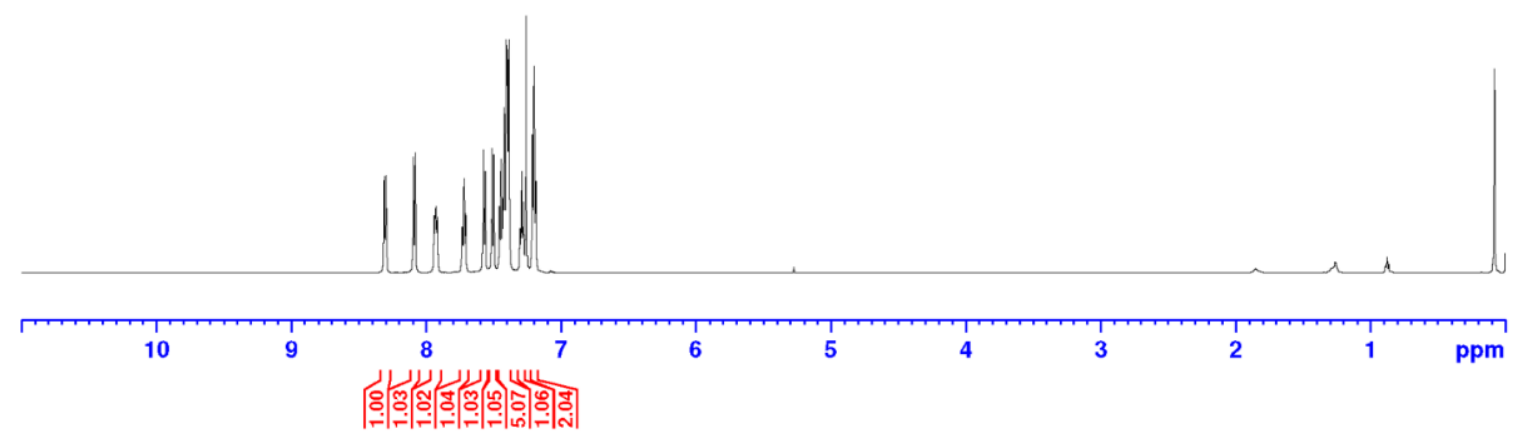

${ }^{1} \mathrm{H}$ NMR of compound $\mathbf{1 0}$ in chloroform- $d$ 
<smiles>O=c1c(-c2ccc3cc(F)ccc3n2)c(-c2ccccc2)oc2ccccc12</smiles>

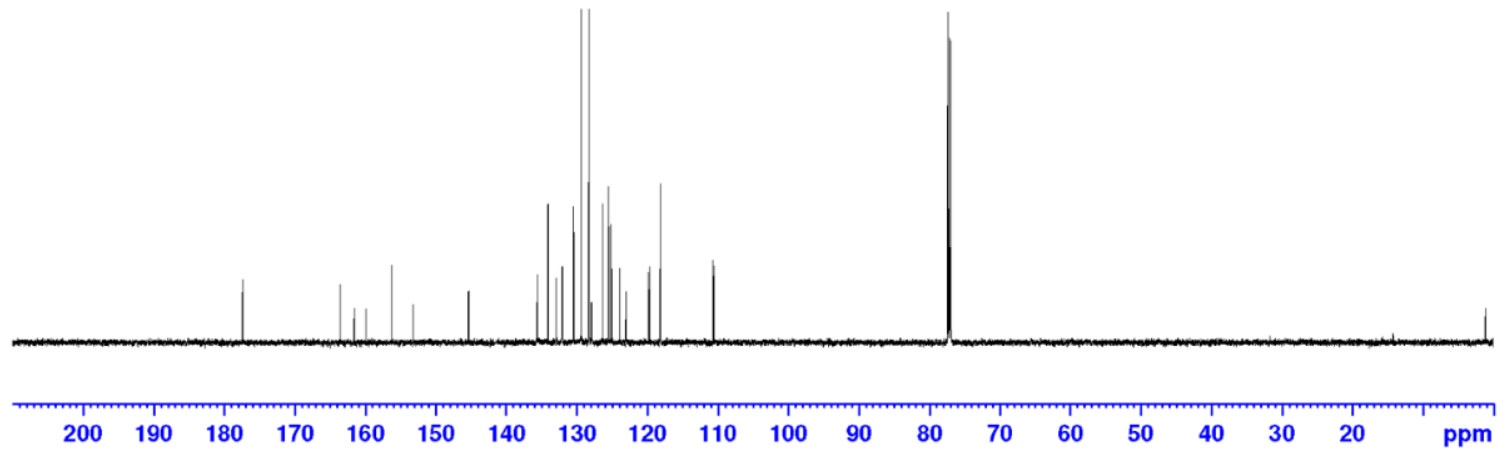

${ }^{13} \mathrm{C}$ NMR of compound $\mathbf{1 0}$ in chloroform- $d$

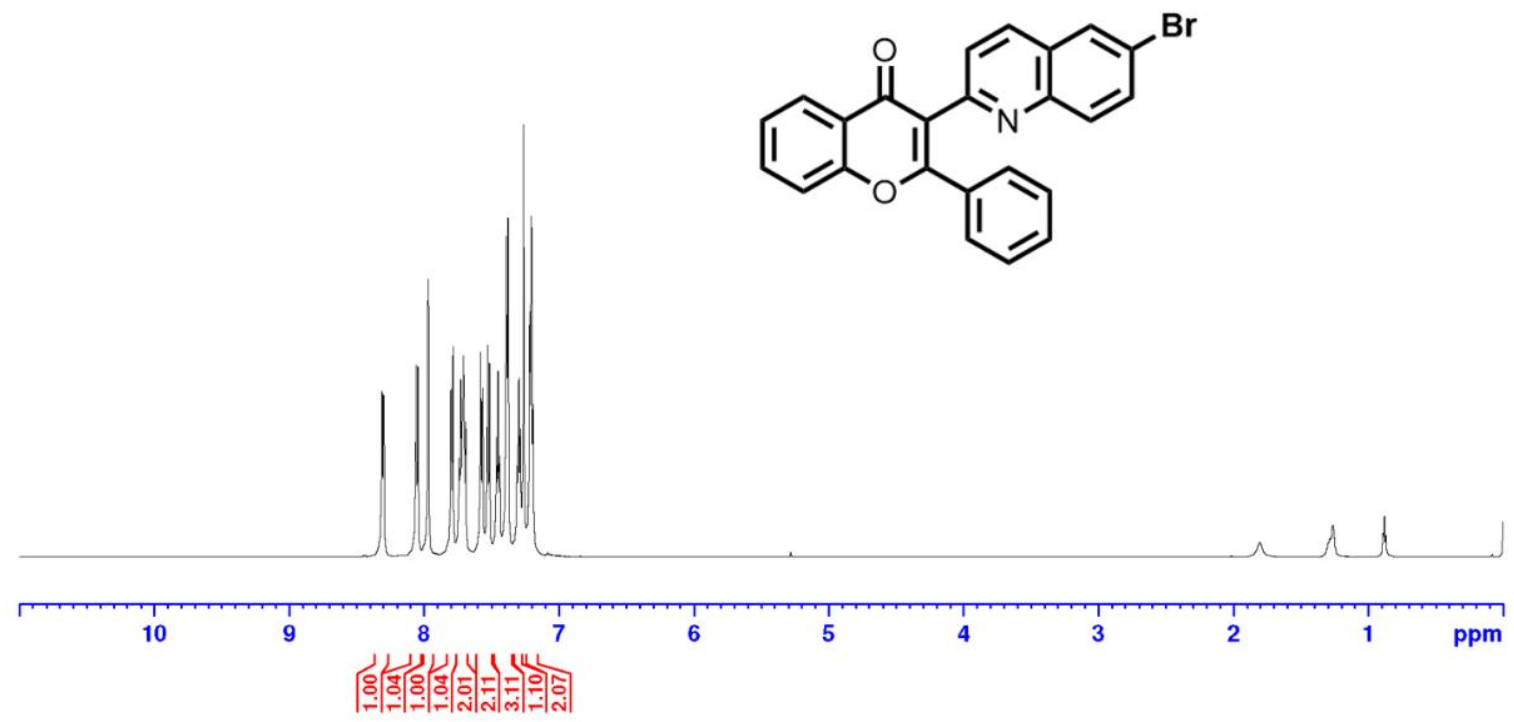

${ }^{1} \mathrm{H}$ NMR of compound $\mathbf{1 2}$ in chloroform- $d$ 


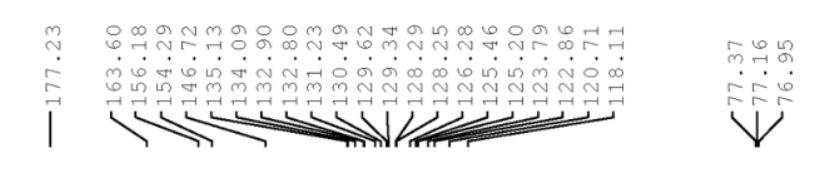<smiles>O=c1c(-c2ccc3cc(Br)ccc3n2)c(-c2ccccc2)oc2ccccc12</smiles>

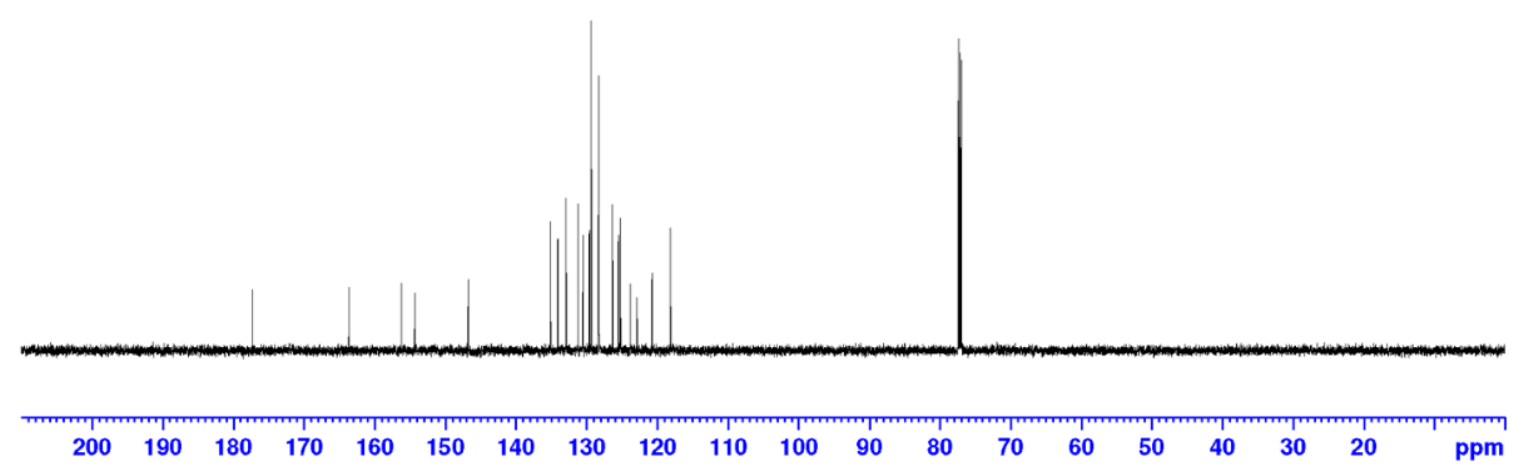

${ }^{13} \mathrm{C}$ NMR of compound 12 in chloroform- $d$
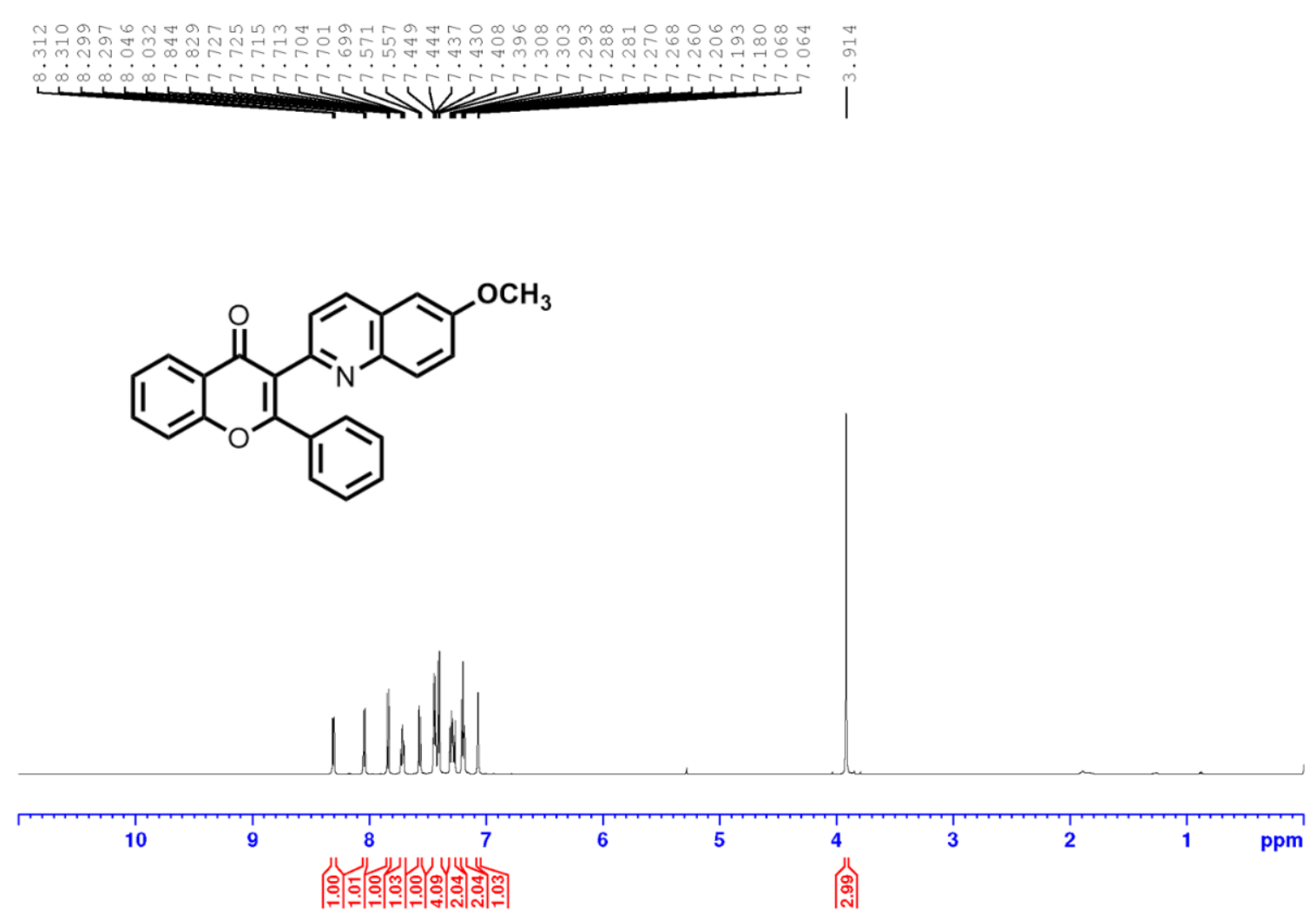

${ }^{1} \mathrm{H}$ NMR of compound $\mathbf{1 3}$ in chloroform- $d$ 

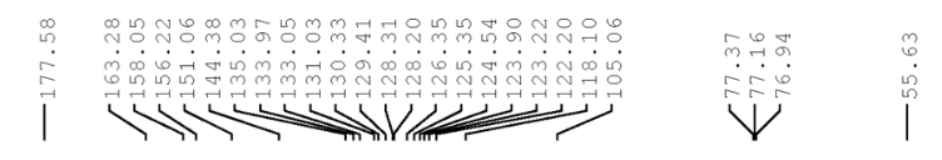<smiles>COc1ccc2nc(-c3c(-c4ccccc4)oc4ccccc4c3=O)ccc2c1</smiles>
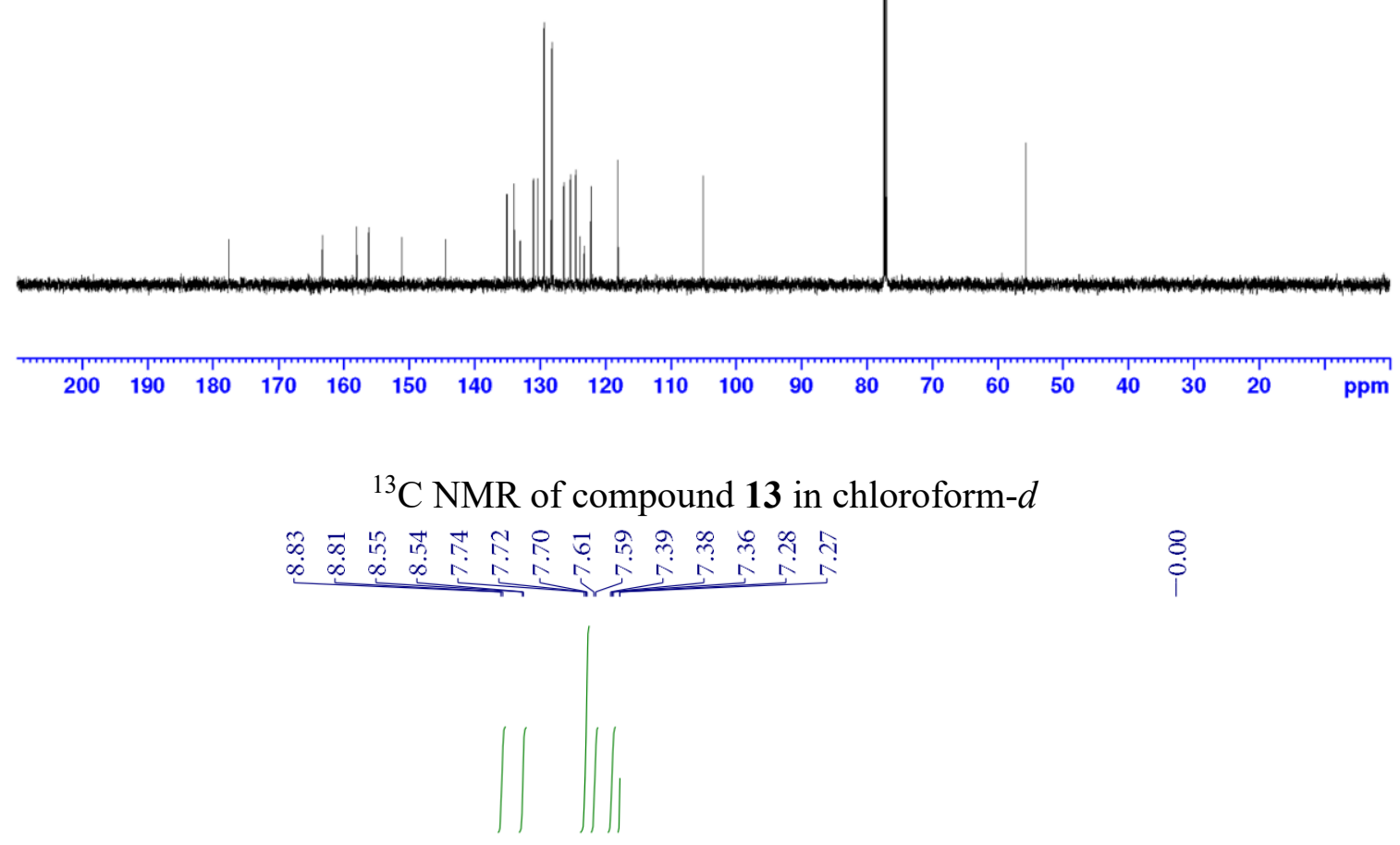<smiles>O=c1c(-c2ccc3cc(OC(F)(F)F)ccc3n2)c(-c2ccccc2)oc2ccccc12</smiles>

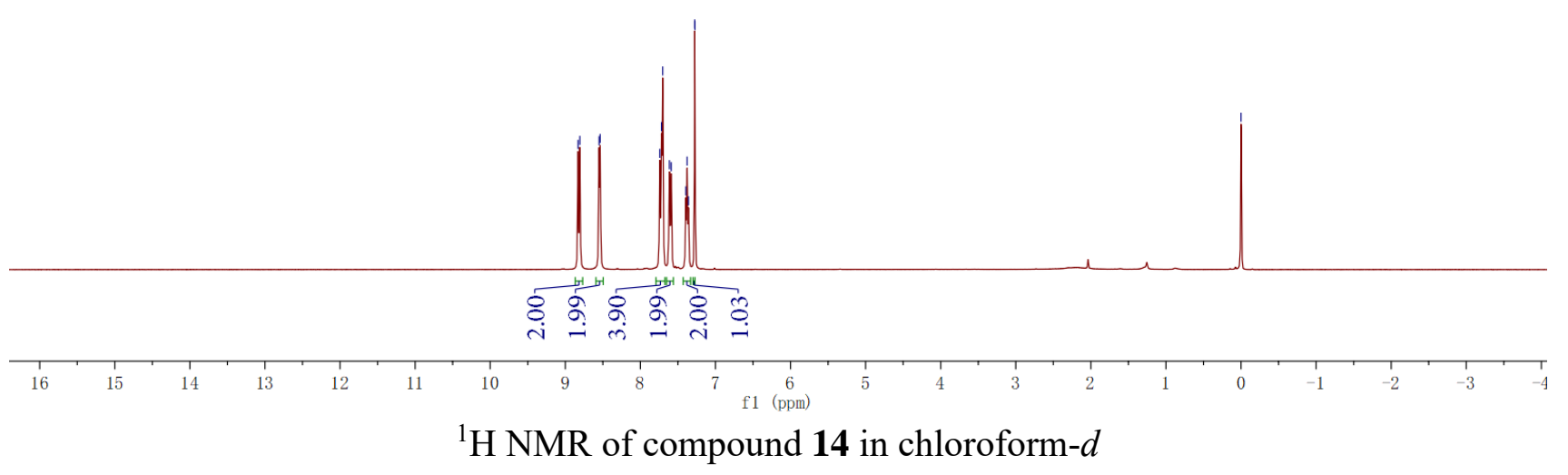



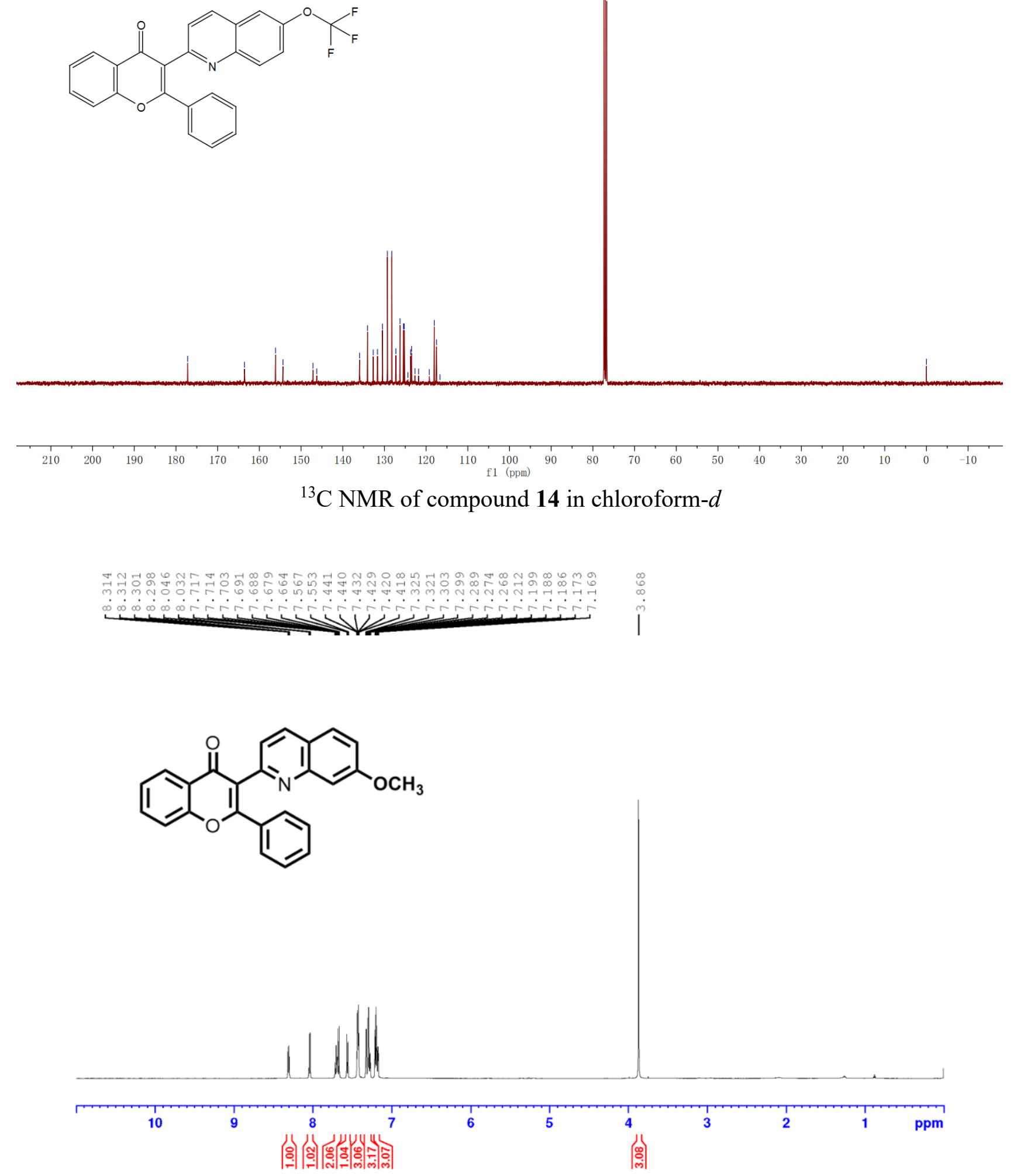

${ }^{1} \mathrm{H}$ NMR of compound $\mathbf{1 5}$ in chloroform- $d$ 

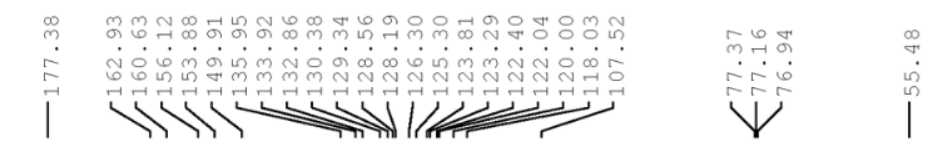<smiles>COc1ccc2ccc(-c3c(-c4ccccc4)oc4ccccc4c3=O)nc2c1</smiles>

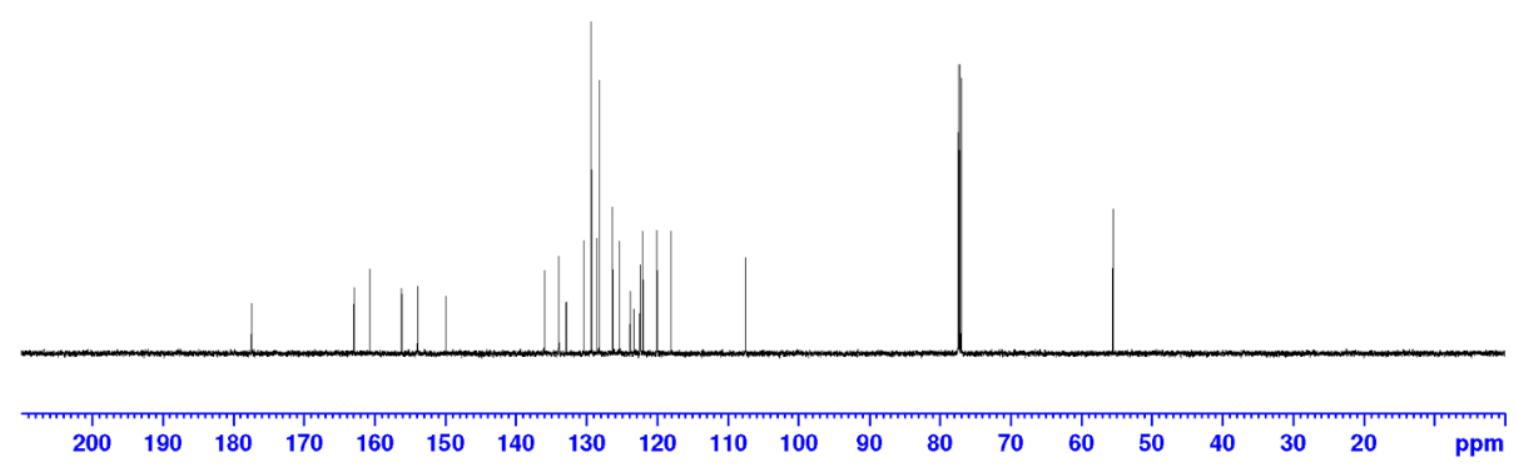

${ }^{13} \mathrm{C}$ NMR of compound $\mathbf{1 5}$ in Chloroform- $d$
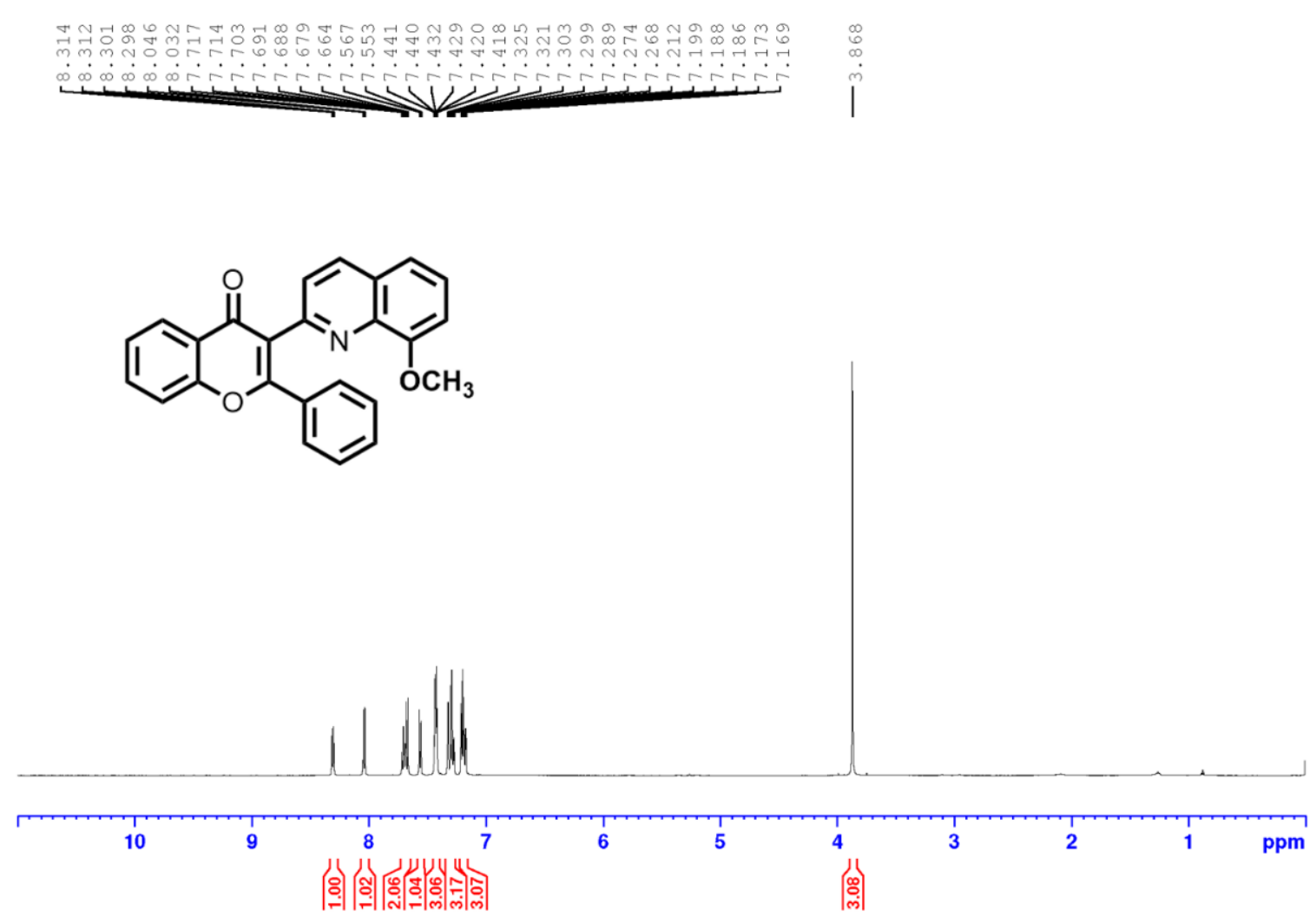

${ }^{1} \mathrm{H}$ NMR of compound $\mathbf{1 6}$ in chloroform- $d$ 

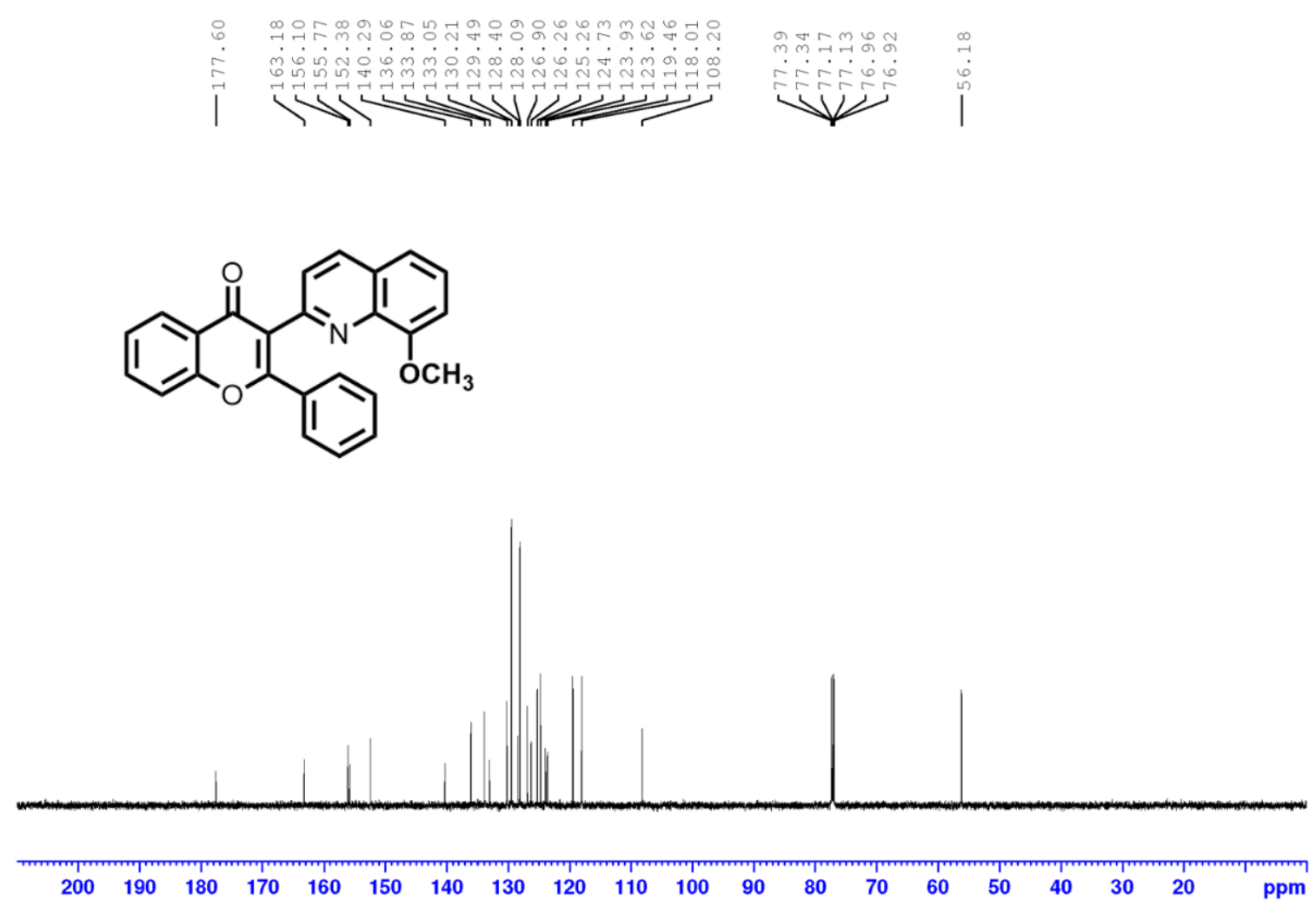

${ }^{13} \mathrm{C}$ NMR of compound $\mathbf{1 6}$ in chloroform- $d$

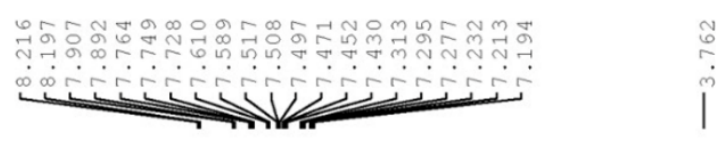<smiles>COc1cc2ccccc2nc1-c1c(-c2ccccc2)oc2ccccc2c1=O</smiles>

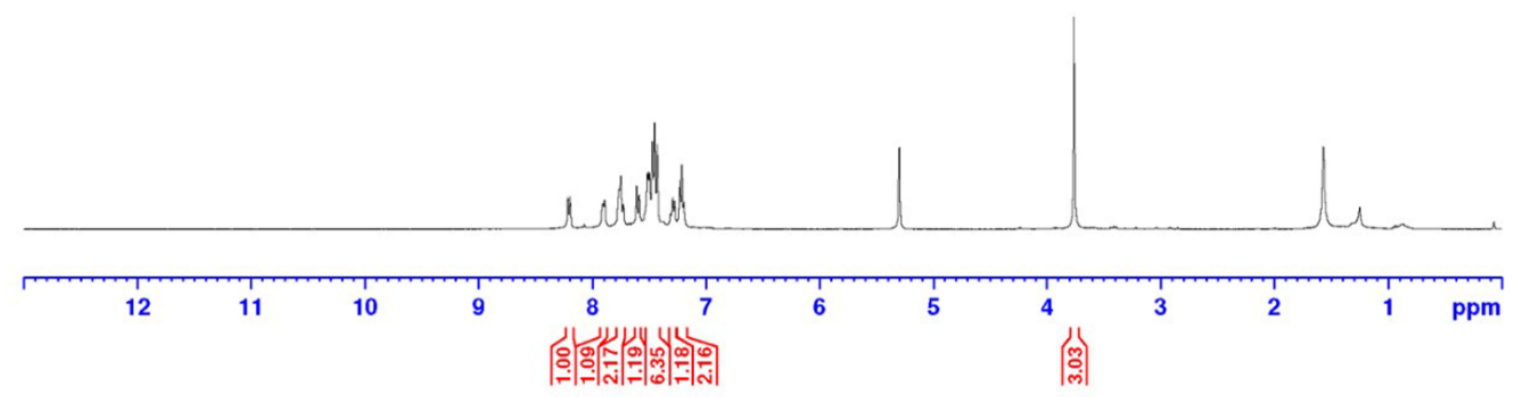

${ }^{1} \mathrm{H}$ NMR of compound $\mathbf{1 7}$ in $\mathrm{CD}_{2} \mathrm{Cl}_{2}$ 


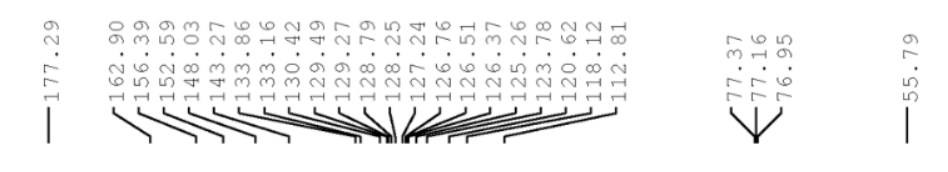<smiles>COc1cc2ccccc2nc1-c1c(-c2ccccc2)oc2ccccc2c1=O</smiles>

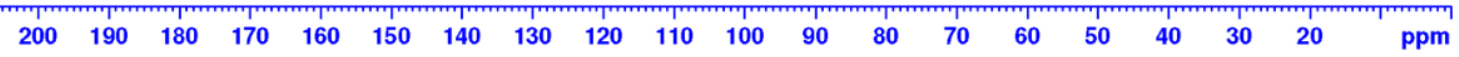

${ }^{13} \mathrm{C}$ NMR of compound $\mathbf{1 7}$ in chloroform- $d$
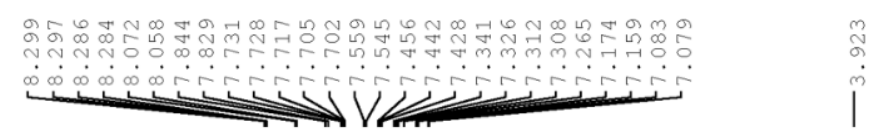<smiles>COc1ccc2nc(-c3c(-c4ccc(Cl)cc4)oc4ccccc4c3=O)ccc2c1</smiles>

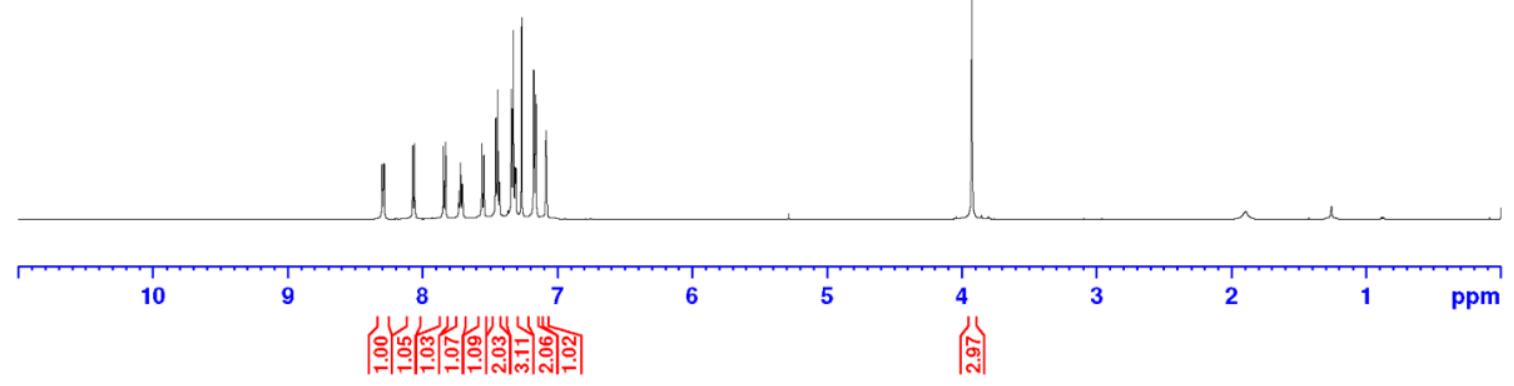

${ }^{1} \mathrm{H}$ NMR of compound $\mathbf{1 8}$ in chloroform- $d$ 

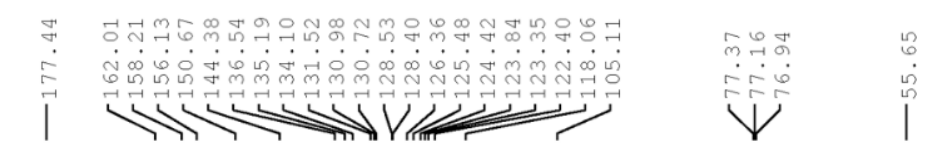<smiles>COc1ccc2nc(-c3c(-c4ccc(Cl)cc4)oc4ccccc4c3=O)ccc2c1</smiles>

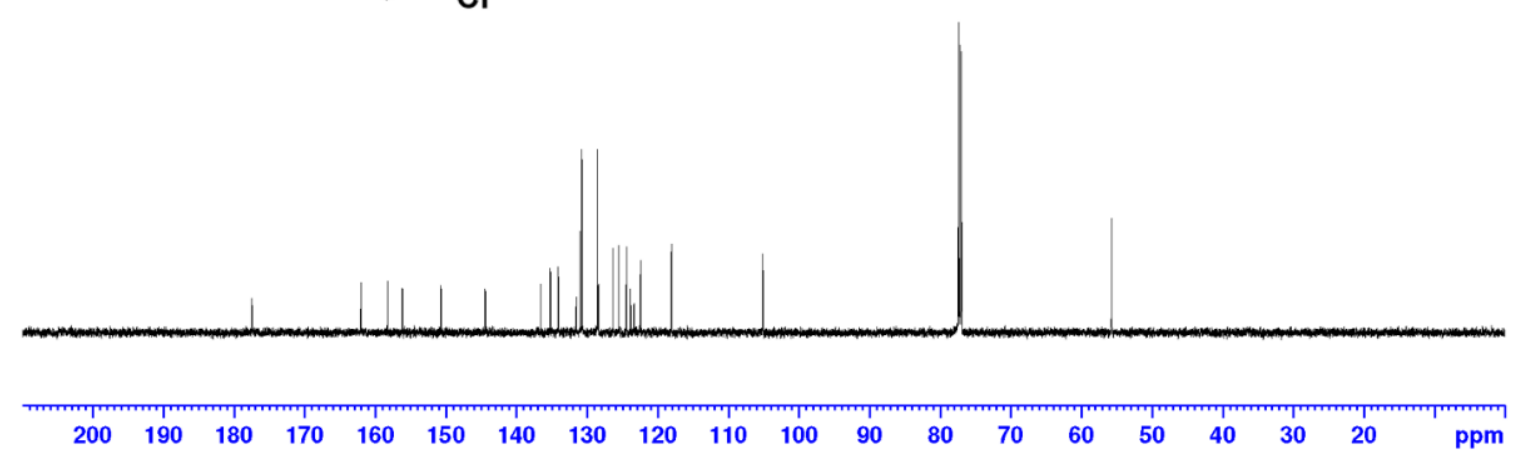

${ }^{13} \mathrm{C}$ NMR of compound $\mathbf{1 8}$ in chloroform- $d$

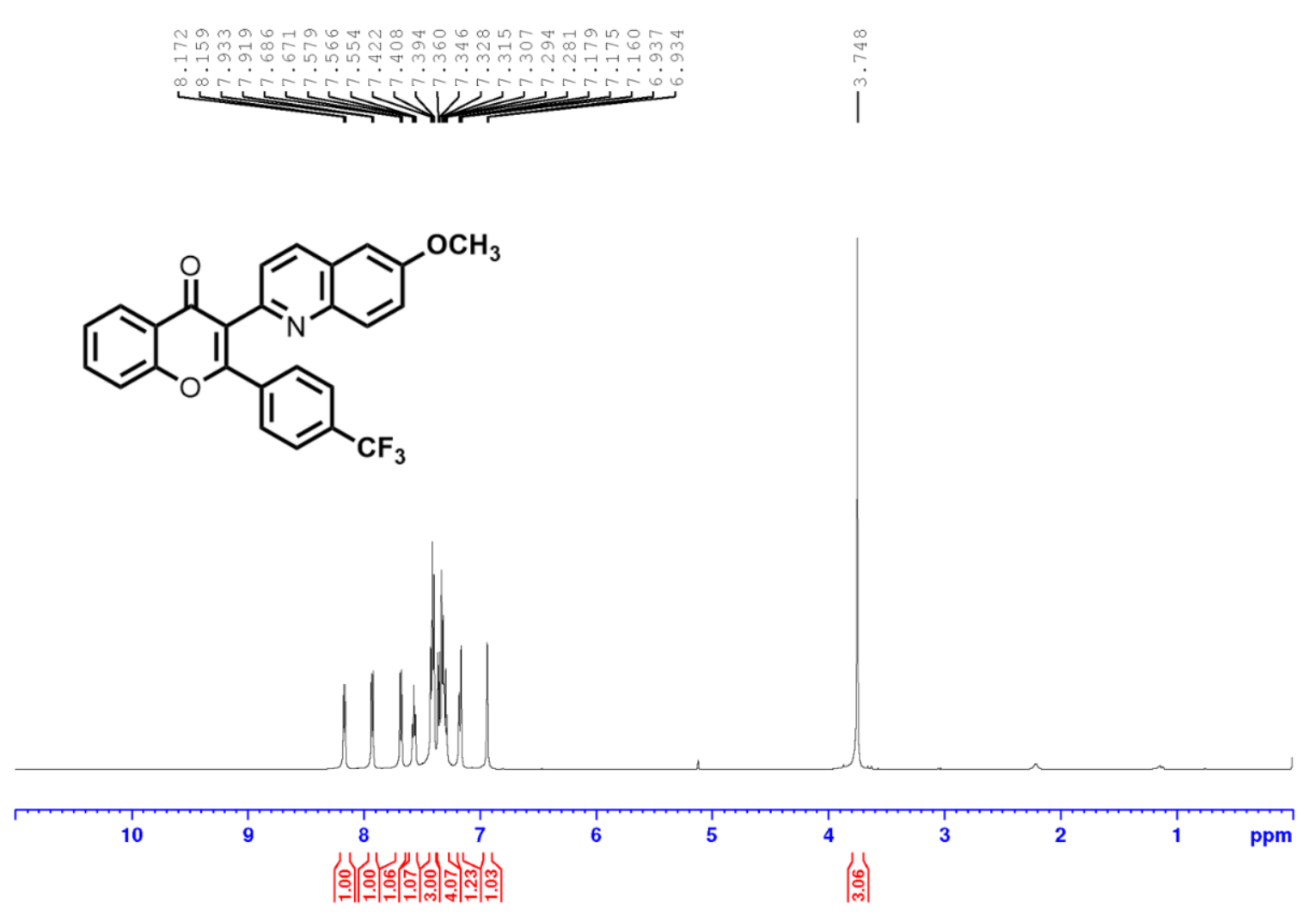

${ }^{1} \mathrm{H}$ NMR of compound 19 in chloroform- $d$ 
<smiles>COc1ccc2nc(-c3c(-c4ccc(C(F)(F)F)cc4)oc4ccccc4c3=O)ccc2c1</smiles>

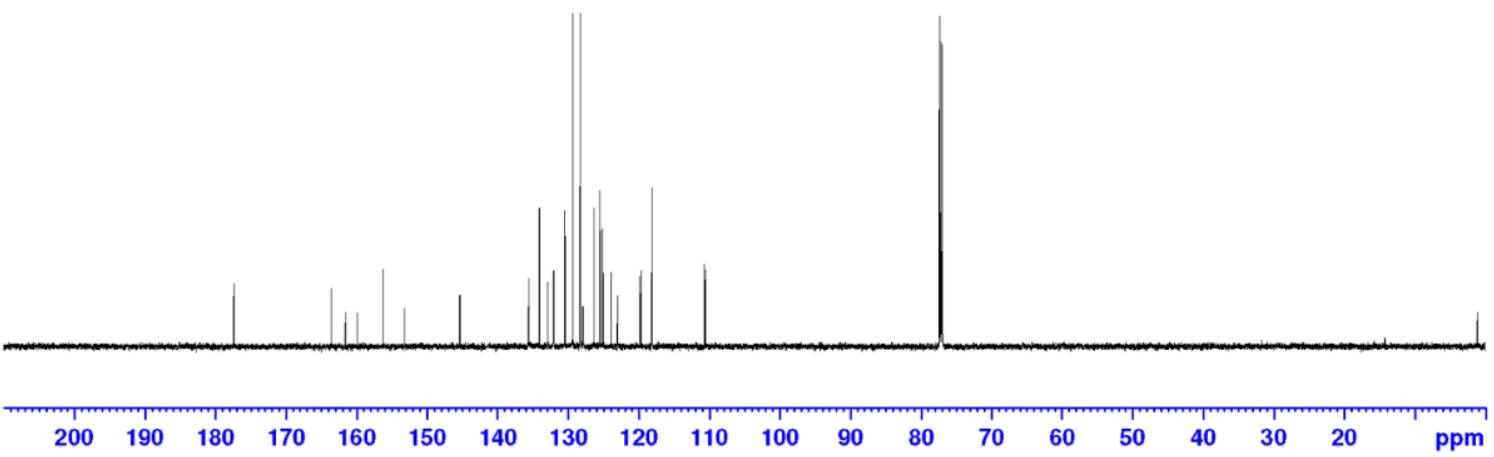

${ }^{13} \mathrm{C}$ NMR of compound 19 in chloroform- $d$<smiles>COc1ccc(-c2oc3ccccc3c(=O)c2-c2ccc3cc(OC)ccc3n2)cc1</smiles>

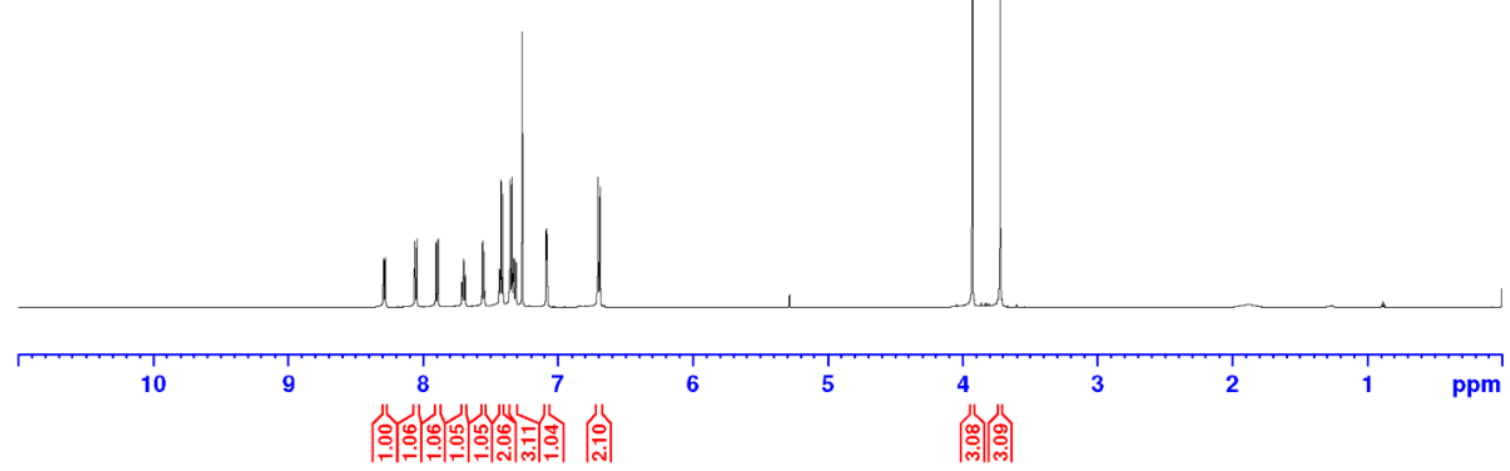

${ }^{1} \mathrm{H}$ NMR of compound $\mathbf{2 0}$ in chloroform- $d$ 
<smiles>COc1ccc(-c2oc3ccccc3c(=O)c2-c2ccc3cc(OC)ccc3n2)cc1</smiles>
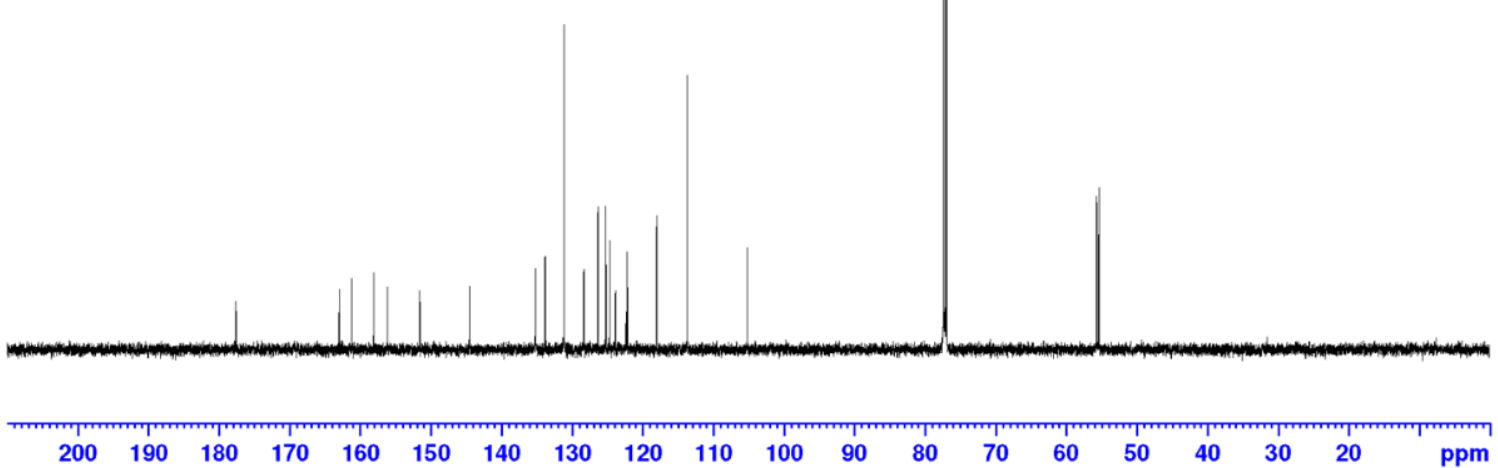

${ }^{13} \mathrm{C}$ NMR of compound 20 in chloroform- $d$
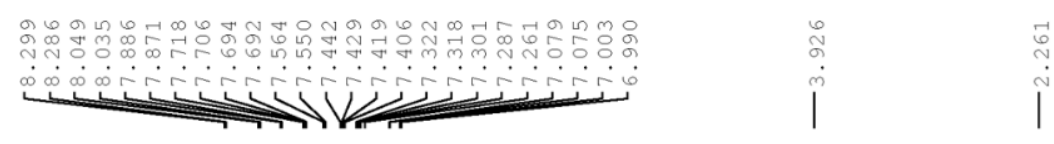<smiles>COc1ccc2nc(-c3c(-c4ccc(C)cc4)oc4ccccc4c3=O)ccc2c1</smiles>

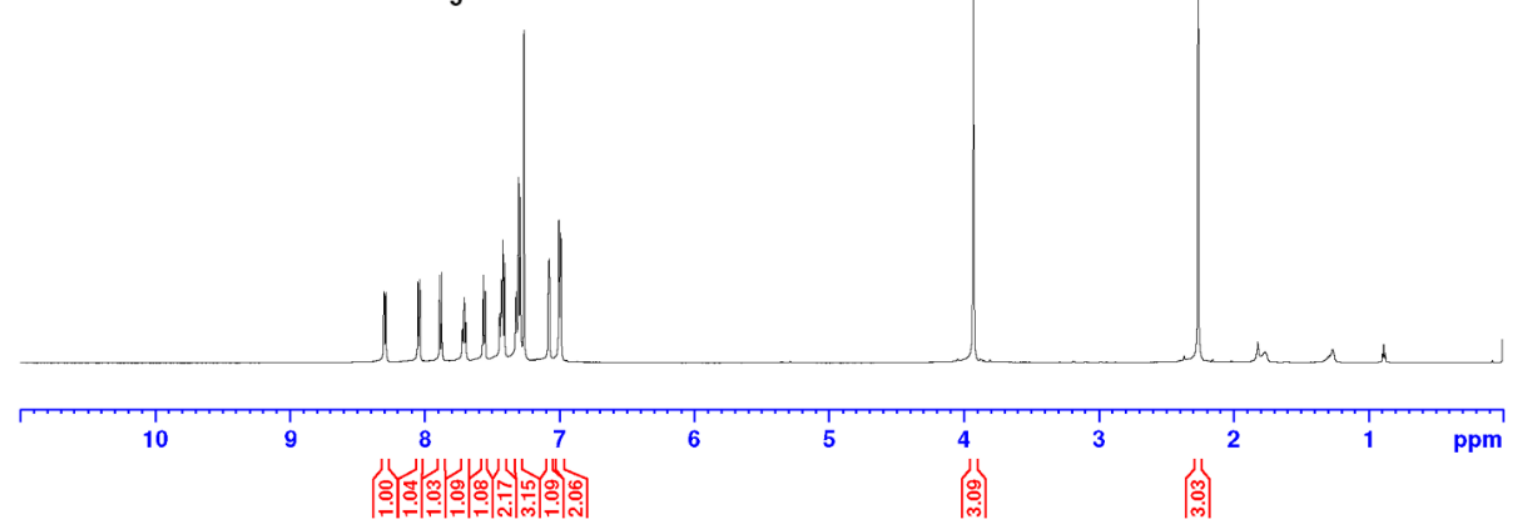

${ }^{1} \mathrm{H}$ NMR of compound 21 in chloroform- $d$ 

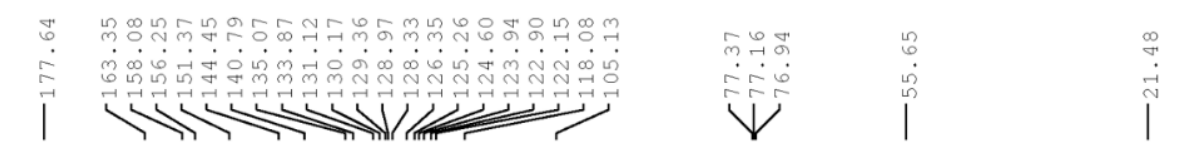<smiles>COc1ccc2nc(-c3c(-c4ccc(C)cc4)oc4ccccc4c3=O)ccc2c1</smiles>

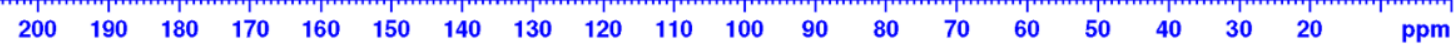

${ }^{13} \mathrm{C}$ NMR of compound 21 in chloroform- $d$

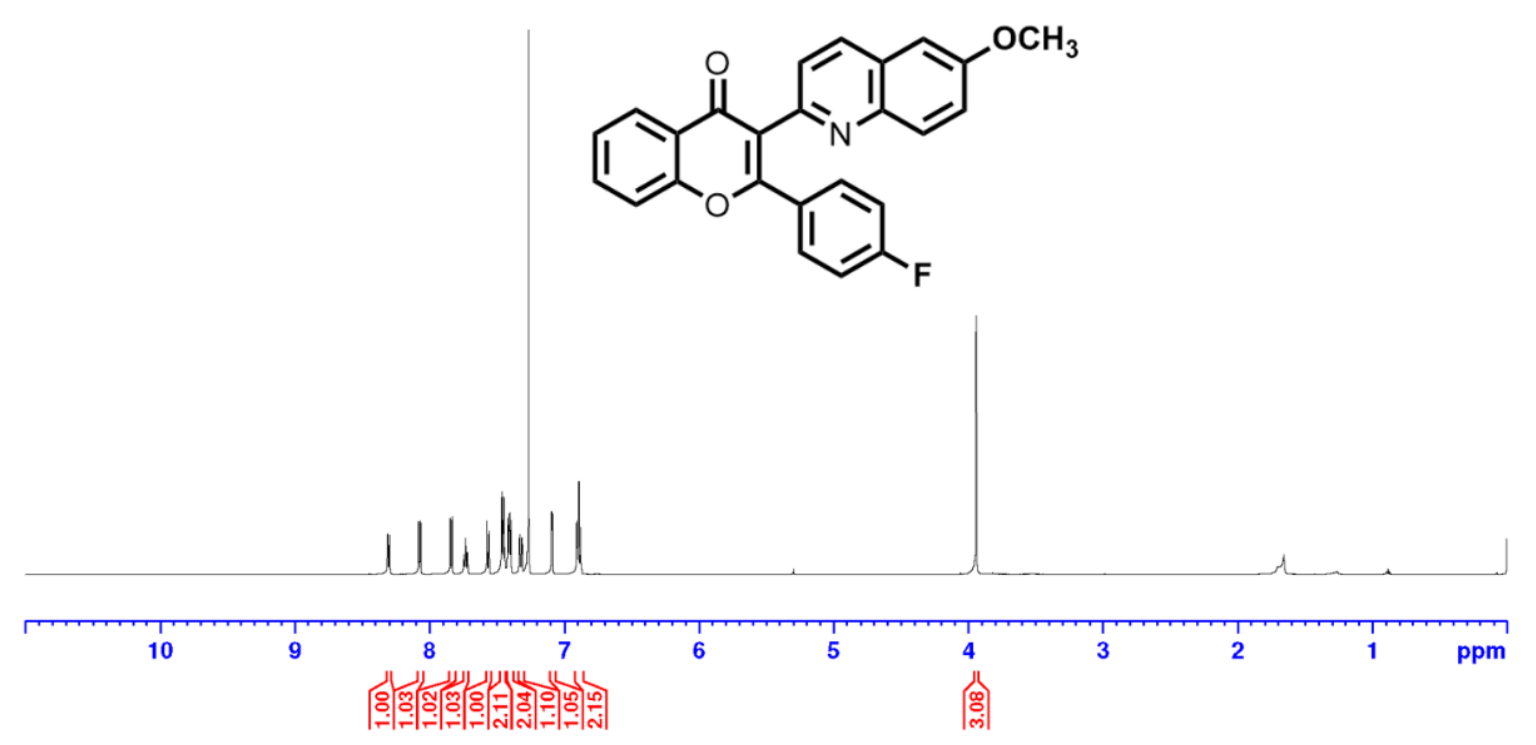

${ }^{1} \mathrm{H}$ NMR of compound 22 in chloroform- $d$ 


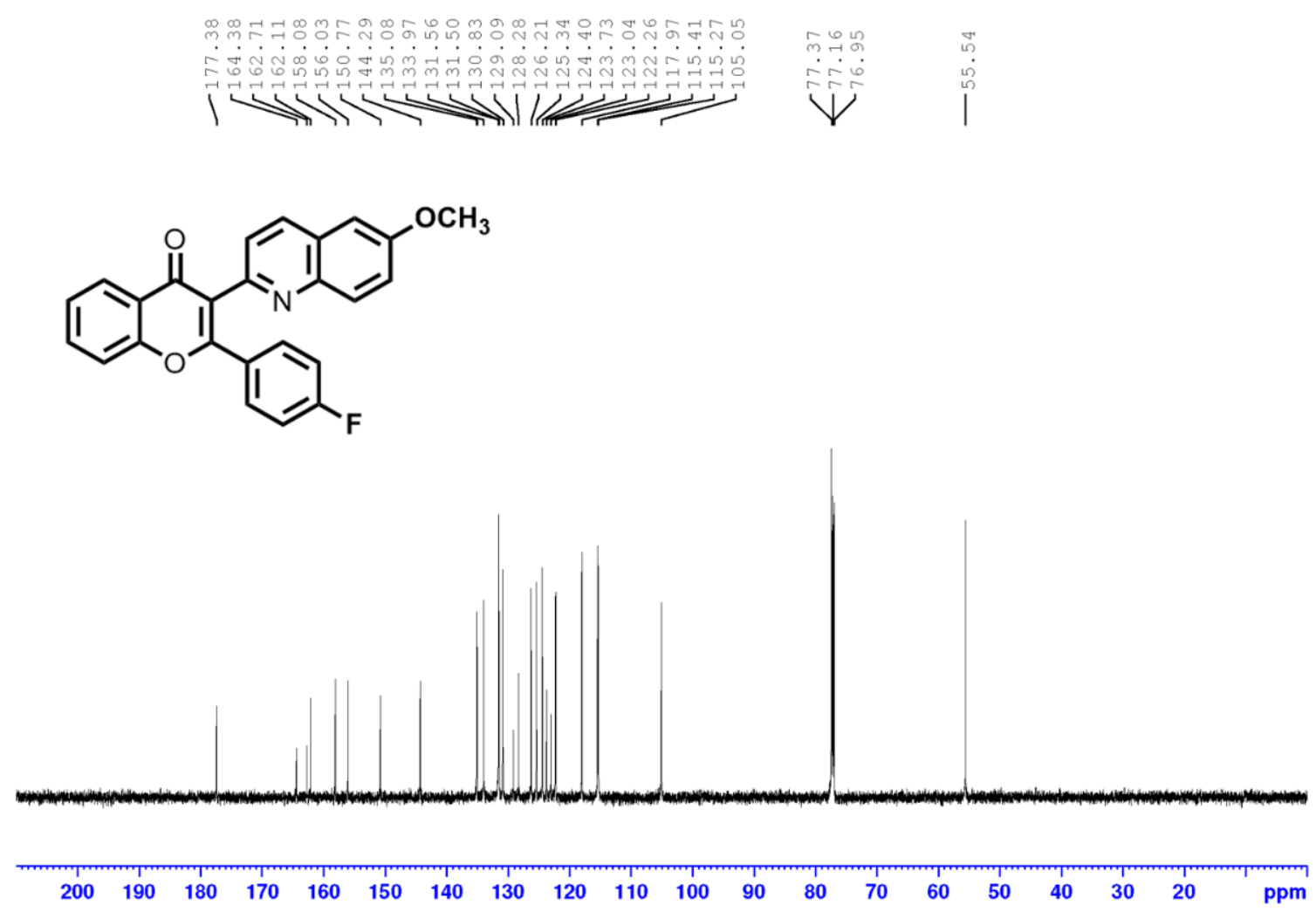

${ }^{13} \mathrm{C}$ NMR of compound 22 in chloroform- $d$ 